\title{
Dorsolateral Prefrontal Lesions and Oculomotor Delayed-Response Performance: Evidence for Mnemonic "Scotomas"
}

\author{
Shintaro Funahashi, a Charles J. Bruce, and Patricia S. Goldman-Rakic \\ Section of Neurobiology, Yale University School of Medicine, New Haven, Connecticut 06510
}

The spatial memory functions of the monkey's prefrontal cortex were examined with oculomotor delayed-response (ODR) paradigms that required the animal to remember the spatial location of peripheral visual cues, while maintaining fixation on a central visual target during the presentation of each cue and during a subsequent $1.5-8$ sec delay period. Four rhesus monkeys received unilateral or serial prefrontal lesions in and around the principal sulcus after they reached criterion performance on the ODR tasks. Unilateral lesions disrupted the performance of memory-guided eye movements to spatial cues in the visual field contralateral to the hemisphere in which the lesion was placed. Memory-guided eye movements to ipsilateral cues were mildly affected by unilateral lesions, and these lesions had fittle or no effect on performance in visually guided control tasks. With addition of a second lesion in the opposite hemisphere, the deficit was extended to include the opposite hemifield. The impairment was characterized by eye movements of inappropriate direction, and, excepting the one lesion that extended into the frontal eye field region of the arcuate sulcus, saccadic reaction times and velocities were the same before and after the lesions. The effect of the lesions was delay dependent: performance was rarely altered at the shortest $(1.5 \mathrm{sec})$ delay but became progressively worse as the delay period was lengthened.

The present results strengthen the evidence that the delayed-response deficits of monkeys with prefrontal lesions are caused by failure to maintain a transient memory "trace" in working memory, and indicate for the first time that working memory mechanisms are lateralized: memories for visuospatial coordinates in each hemifield are processed primarily in the contralateral prefrontal cortex. These findings provide evidence for the concept of mnemonic hemianopias and mnemonic scotomas, that is, memory deficits for particular hemifields or visual field locations, unaccompanied by simple sensory or motor deficits.

[Key words: prefrontal cortex, monkey, cortical lesion, spatial memory, mnemonic scotomas, oculomotor delayedresponsel

\footnotetext{
Received July 27, 1992; revised Oct. 1, 1992; accepted Oct. 6, 1992.

This work was supported by National Institute of Mental Health Grants MH38546 and MH-00298 (P.S.G.-R.) and National Eye Institute Grant EY-04740 (C.J.B.). We thank G. Leydon for programming assistance, M. Papero and S. Morgenstern for surgical assistance and animal care, J. Coburn and M. Pappy for histology, and J. Musco for photography.

Correspondence should be addressed to Dr. P. S. Goldman-Rakic, Section of Neurobiology, Yale University School of Medicine, 333 Cedar Street, New Haven, CT 06510-8001.

a Present address: Department of Health and Exercise Science, College of Liberal Arts and Sciences, Kyoto University, Sakyo-ku, Kyoto 606, Japan.

Copyright (C) 1993 Society for Neuroscience $0270-6474 / 93 / 131479-19 \$ 05.00 / 0$
}

The integrity of the dorsolateral prefrontal cortex [Walker's area 46 (Walker, 1940)] is essential for performance on delayedresponse tasks, which engage working memory. Since Jacobsen first described that monkeys with bilateral lesions in the prefrontal cortex are profoundly impaired in performance on delayed-response tasks (Jacobsen, 1935), the association between the delayed-response task and the prefrontal cortex has been confirmed by a wide variety of techniques [see reviews by Fuster (1989) and Goldman-Rakic (1987)]. In addition, the minimal lesion site necessary to produce the classical delayed-response deficit has been delimited to the cortex lining the principal sulcus (PS) (Gross, 1963; Butters and Pandya, 1969; Goldman and Rosvold, 1970; Goldman et al., 1971; Butters et al., 1972; Rosenkilde, 1979).

Although there is good agreement on the cortical area involved in delayed-response performance, there are a number of different interpretations on the nature of the deficit. Originally, Jacobsen interpreted the delayed-response impairment as a loss of "immediate" memory (Jacobsen, 1935), but this view of prefrontal function was not widely accepted (see Goldman-Rakic, 1990, for further discussion of this point). Subsequent investigators advanced alternative hypotheses, including a deficit in attentional (Bartus and Levere, 1977), inhibitory (Luria, 1966), or perceptual (Teuber, 1972) mechanisms.

Difficulties in interpreting the psychological processcs tapped by the delayed-response task can be attributed partly to the instruments used to examine this task. The classical delayedresponse task has usually been administered to unrestrained monkeys in a Wisconsin Test Apparatus, in which the monkey can view the behaviorally relevant stimuli from a variety of angles and is free to move around the cage during the delay and response periods. Although monkeys with appropriate prefrontal lesions consistently fail the delayed-response task, limitations in manipulation of the number and position of stimuli, the activities of the animal in the delay period, and other parameters in this task have made it difficult to assess where the lesioned monkey's difficulty lay.

In the present experiment, we used an oculomotor analog of the classical manual delayed-response task to examine the spatial memory function of the prefrontal cortex under conditions permitting relatively precise control over the monkey's behavior. The design of this oculomotor delayed-response (ODR) task allowed us to present cues at multiple locations in the visual field, to control their retinotopic locations, to prevent postural orientation during the delay period that could negate the mnemonic requirement, and also to analyze the monkey's behavioral responses quantitatively (accuracy, latency, direction, amplitude, duration, etc.). We have employed this exact task extensively to examine the functional properties of prefrontal neurons 
A. Oculomotor delayed-response ( (ODR-I)

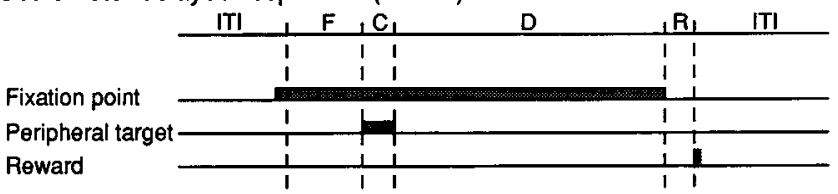

B. Oculomotor delayed-response II (ODR-II)

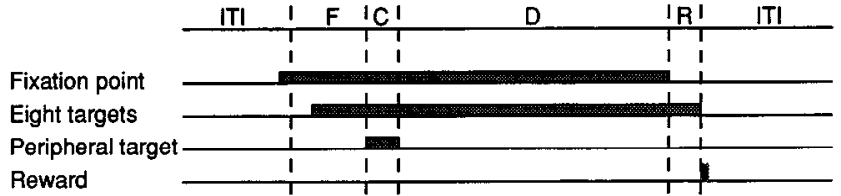

C. Control task I (delayed visually guided saccade)

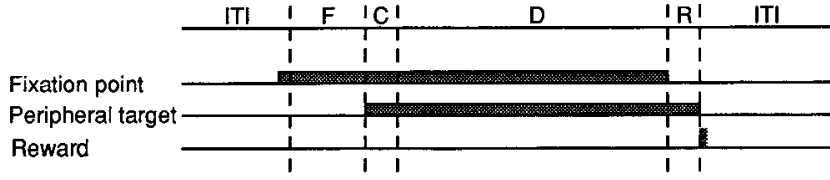

D. Control task II (visually guided saccade)

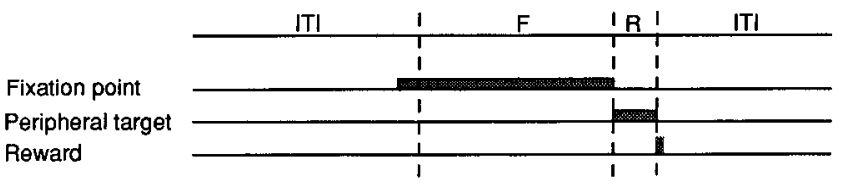

Figure 1. Schematic drawings of the behavioral paradigms. $A$, ODR-I task. $B$, ODR-II task. $C$, Delayed visually guided saccade task (control task I). $D$, Visually guided saccade task (control task II). Horizontal and vertical eye position, timing of visual stimulus presentations (fixation target, visual cue, saccade target), and reward delivery are shown for each task. Abbreviations: $I T I$, intertrial interval; $F$, fixation period; $C$, cue period; $D$, delay period; $R$, response period.

(Funahashi et al., 1989, 1990, 1991), and similar ODR tasks have been used to study neurons in several other brain structures. Most recently, the ODR paradigm has been adapted to the study of prefrontal function in schizophrenic patients (Park and Holzman, 1992).

\section{Materials and Methods}

\section{Subjects}

Four adult rhesus monkeys (Macaca mulatta, $3.2-6.5 \mathrm{~kg}$ ) served as subjects. Among them, two monkeys (KY and DN) had been used for single-neuron recording studies (Funahashi et al., 1989, 1990) before prefrontal cortical lesions were made. The monkeys received their daily ration of water $(150-300 \mathrm{ml})$ in the testing chamber and were given monkey chow immediately on return to their home cages. Their intake of food and water, and body weight were closely monitored.

\section{Behavioral testing apparatus}

Behavioral training and testing sessions were conducted daily and usually lasted $2-4 \mathrm{hr}$. The monkeys sat in a primate chair in the dark during testing, and their heads were fixed to the chair by a restraining receptacle. A program on a PDP-11 computer presented the visual stimuli and delivered rewards. Visual stimuli were presented on a monochrome cathode ray tube (CRT) (19 inch, RCA TC1119). The fixation target was a small white spot $\left(0.1^{\circ}\right.$ diameter $)$, presented at the center of the $\mathrm{CRT}$, and the peripheral visual cues were usually white squares $\left(0.7^{\circ} \times\right.$ $0.7^{\circ}$ ). There was no background illumination on the CRT. The monkeys were rewarded with a drop $(0.2 \mathrm{ml})$ of lightly sweetened water delivered by an electronic metering pump (Waltham A741).

Eye movements were recorded by a magnetic search coil technique (Robinson, 1963). The monkey's oculomotor behavior was monitored during the experiments using computer-generated displays on a digital oscilloscope (Hewlett-Packard 1345A). Horizontal gaze, vertical gaze, and overall eye velocity were displayed as scrolling traces. Two-dimensional representations of gaze, visual stimuli, and the fixation window were superimposed on the scrolling display.

\section{Behavioral paradigms}

The effects of lesions in the principal sulcus and surrounding prefrontal cortex were examined on performance on two versions of the oculomotor task-ODR-I or ODR-II-and under one or more of the control tasks described below. ODR tasks included three different delay period durations $(1.5,3$, and $6 \mathrm{sec})$. In order to use each monkey as its own control, the monkey's performance was examined both before and after surgery and, for two monkeys, also after a second prefrontal lesion made in the other hemisphere.

Oculomotor delayed-response I (ODR-I) (Fig. 1A). All four monkeys were trained and tested on ODR-I. After a $5 \mathrm{sec}$ intertrial interval, the fixation target appeared at the center of the CRT and the monkey was required to maintain fixation for $0.75 \mathrm{sec}$ before the visual cue was presented for $0.5 \mathrm{sec}$ (the cue period) at one of eight peripheral locations. The location of these stimuli was randomized over trials so that the monkey could not predict where the cue would appear on any given trial. A crucial feature of the task is that the monkey was required to maintain fixation throughout the cue and the subsequent $1.5-8$ sec delay periods. At the end of the delay period, extinction of the fixation point signaled the animal to initiate a response. A response was defined as correct if it occurred within $0.5 \mathrm{sec}$ of fixation point offset (the response period) and also fell within a $5^{\circ}$ radius of the cue location. Correct responses were rewarded with a drop of juice; errors were not rewarded.

Stimuli were presented at one of eight peripheral cue locations separated by $45^{\circ}$ in polar direction. The eccentricity of each cue was $13^{\circ}$ in visual angle from the central fixation target. The assignment of three different delay durations (usually $1.5,3$, and $6 \mathrm{sec}$ ) across cue locations $\left(0^{\circ}, 45^{\circ}, 90^{\circ}, 135^{\circ}, 180^{\circ}, 225^{\circ}, 270^{\circ}\right.$, and $\left.315^{\circ}\right)$ yielded a total of 24 delay $/$ cue combinations. Each session usually consisted of blocks of 400 or more trials so that each direction/delay combination occurred randomly at least 15 times.

Oculomotor delayed-response II (ODR-II) (Fig. 1B). Three of the four monkeys in the study were also tested on the ODR-II variant of the task. The main difference between ODR-I and ODR-II is that in the latter, eight small targets remained on the CRT during the delay and response periods, providing a spatial reference but no information about the correct cue on any trial. The same combinations of delay and cue locations were used in this task as in the ODR-I task, and again the location of the visual cue was randomized over trials.

Control task I (delayed visually guided saccades) (Fig. 1C). The stimulus sequence, cue locations, delay durations, and the required eye movements were exactly the same as those of the ODR-I task (compare Fig. $1 C$ with $1 A$ ). The only difference between them was that in the control task the visual cue remained on throughout the delay and response periods. Thus, in this task, the saccade at the end of the delay was sensory guided, that is, directed by a single visible target. As in the ODR tasks, the monkey was required to make a saccade within the 0.5 sec response period.

Control task II (visually guided saccades) (Fig. 1D). After the monkey maintained fixation for $3 \mathrm{sec}$, the fixation target disappeared and, simultaneously, a peripheral visual target was presented in one of the eight locations. The monkey was simply required to make a saccadic eye movement to the peripheral target within the $0.5 \mathrm{sec}$ response period. Again, this is a sensory-guided task. The locations and dimensions of the peripheral visual stimuli and the size of the response window were the same as in all other tasks.

\section{Training procedures}

The monkey was initially trained to simply fixate a central visual target. After it achieved fixation for 4-6 sec, a peripheral visual cue was introduced at one of the eight locations for $0.5 \mathrm{sec}$ in the middle of the fixation period. At first, the monkey was required to maintain fixation and ignore the visual cue. Then, a second visual cue was presented at the same location as that of the first cue after the end of the fixation 
period, and the monkey was required to make a saccadic eye movement to the second cue within $1.0-1.5 \mathrm{sec}$. After the monkey achieved this task, we fixed the delay period at $0.5 \mathrm{sec}$, used four cue locations $\left(0^{\circ}\right.$, $90^{\circ}, 180^{\circ}$, and $270^{\circ}$ ), and gradually reduced the duration of the second cue presentation to zero. After the monkey performed correct saccades without the second cue, all eight cue locations were introduced and the delay duration was gradually increased from $0.5 \mathrm{sec}$ to $8 \mathrm{sec}$. Approximately 2 months was required for the monkey to complete training on the ODR-I task. Once the monkey learned the ODR-I task, it performed the two control tasks without any additional training; however, an additional month was needed to master the ODR-II task.

\section{Surgical procedures}

To record eye movements, a search coil was placed under the conjunctiva in one eye at surgery. Following adaptation to a primate chair, each monkey was surgically prepared under aseptic conditions and barbiturate anesthesia (pentobarbital sodium) for implantation of the search coil as well as a head bolt. The coil was inserted using the technique of Judge et al. (1980). To secure the implant, stainless steel bolts with flattened heads were run along slots in the skull with the bolt head under the skull. The bolts, a connector for the search coil, and a stainless steel receptacle for attaching the monkey's head to the primate chair were bound together with dental acrylic. Monkeys were given systemic antibiotics, fruits, and ad lib water and chow for 7-10 d following surgery. Once overall performance levels reached $80 \%$ correct or above in both ODR tasks, the monkeys received ablations in and around the principal sulcus by subpial aspiration under barbiturate anesthesia using sterile surgical technique. Two monkeys (KY and DN) received unilateral ablations; each of these two animals had a recording chamber over its other frontal lobe, and we needed to recover electrode tracks and marking lesions and deposits from this hemisphere. Two additional monkeys (YM and JN) were given additional cortical ablations in the opposite prefrontal cortex 1.5 and 2.5 months, respectively, after the first ablation. In one monkey (YM) the frontal eye field (FEF) was deliberately involved. Reconstructions of the extent of the prefrontal lesions for each monkey are shown in the Results. Monkeys were treated with antibiotics and given fruit, water, and chow ad libitum for at least $7 \mathrm{~d}$ following surgery. Postoperative testing lasted $2-8$ months.

\section{Data acquisition and analysis}

A PDP-11 computer, in addition to carrying out the behavioral paradigms, stored three types of data files on magnetic media for later analyses.

Behavior files contained the number of correct and incorrect trials for each direction/delay condition within a session that was used to calculate the proportions of errors in each condition and to generate polar plots of the data (e.g., see Fig. 4).

Event buffer files contained the time of every event that the computer had access to, including the time, duration, and eye position at the start and end of each saccadic eye movement the monkey made, as recognized on line using the algorithm of van Gisbergen et al. (1981). Individual event buffer files usually contained 250-500 trials. Means and standard deviations of the $x$-and $y$-coordinates of the end points of the response period saccades corresponding to each combination of visual cue location and delay period duration were computed and used to plot ellipses that graphically illustrated saccade variability (e.g., see Fig. 6). Similarly, the size of saccade errors was calculated as the distance (in degrees subtended) between the end point of the response period saccade and the location of the cue (e.g., see Fig. 8). Saccadic reaction time was defined as the time interval between the disappearance of the fixation target and the initiation of the response period saccade (e.g., see Fig. 19).

Analog buffer files contained multiple records (1-2 sec epochs preceding and following the saccade) of all the analog signals being sampled at $4 \mathrm{msec}(250 \mathrm{~Hz})$, together with a code representing progress through the task paradigm. Using analog buffer files, we compared trajectories of saccadic eye movements, before and after the lesions, in the different delay and direction conditions and across different tasks.

\section{Histological examination}

After all behavioral testing was completed, the monkeys were killed by injecting an overdose of pentobarbital sodium and perfused with an initial flush of $0.9 \%$ sodium chloride solution through the heart, followed by $10 \%$ formalin fixative. A graded series of $5 \%, 10 \%$, and $15 \%$ sucrose
Table 1. Summary of prefrontal ablations

\begin{tabular}{lll} 
Monkey & $\begin{array}{l}\text { First lesion } \\
\text { (left hemisphere) }\end{array}$ & $\begin{array}{l}\text { Second lesion } \\
\text { (right hemisphere) }\end{array}$ \\
\hline KY & PS & None \\
JN & PS + DLC & PS + DLC \\
DN & Anterior PS & None \\
YM & Posterior PS + AS & Middle PS \\
\hline
\end{tabular}

PS, Principal sulcus; DLC, dorsolateral convexity; AS, arcuate sulcus (frontal eye field).

formalin solutions were sequentially administered for cryoprotection. The brain was removed from the skull, photographed, and kept in $20 \%$ sucrose formalin until it sank. The frontal lobes were frozen sectioned at $40 \mu \mathrm{m}$ thickness; every fifth section was collected and stained by the Nissl method for later histological examination.

\section{Results}

\section{Overview}

Table 1 summarizes the lesions made in all four monkeys. In all four the initial lesion was placed in the left hemisphere and primarily involved the cortex of the PS. Two monkeys received a subsequent lesion involving PS cortex of the right hemisphere. As summarized in the table, the location and extent of the lesions within PS varied across the six experimental lesions, and in three of the six cases cortex adjacent to the PS was also removed, with one of these involving the arcuate sulcus (AS) where the FEF is located. Reconstructions from each of the four monkeys are presented later in the Results.

Figure 2 summarizes the effect of the initial PS lesion on ODR-I, ODR-II, and control task pcrformance in all four monkeys. In each monkey the initial lesion was in the left hemisphere, and in each monkey a deficit in the performance for some or all cue locations in the right visual field was observed when testing resumed approximately 1 week later. As shown in the figure, performance on the sensory-guided control task was nearly perfect in each case. Two monkeys subsequently received a lesion of right PS, and in both additional testing showed a deficit in performing the ODR task for cue locations in the left visual field, together with some recovery of their right field deficits. Again, performance in control tasks was unimpaired. Thus, the following sections describe the lesions and focus on the performance under the various delay conditions of the ODR task for each of the four monkeys studied.

\section{Case by case analysis of lesions and performance}

Monkey KY. Figure 3 shows the reconstruction of monkey KY's lesion. The lesion extended almost the entire length of PS, and included both its upper and lower banks and fundus. The lesion was restricted to the PS area and no damage was observed in AS posterior to PS or in the cortex of the superior or inferior dorsolateral convexity surrounding the PS.

Preoperatively, monkey KY performed well in the ODR-I task for all eight cue directions under all three delay durations. The left column of Figure 4 shows plots of the percentages of errors made in both the ODR-I and ODR-II tasks during the final testing days before the lesion surgery. The percentage error score's plot on ODR-I (Fig. 4, top left) indicates that this monkey was proficient for most all cue and delay combinations, but had some difficulty with $6 \mathrm{sec}$ delays in the left field, particularly the upper left direction. Similarly, in the ODR-II task it was above $90 \%$ correct for most cue and delay combinations, but 

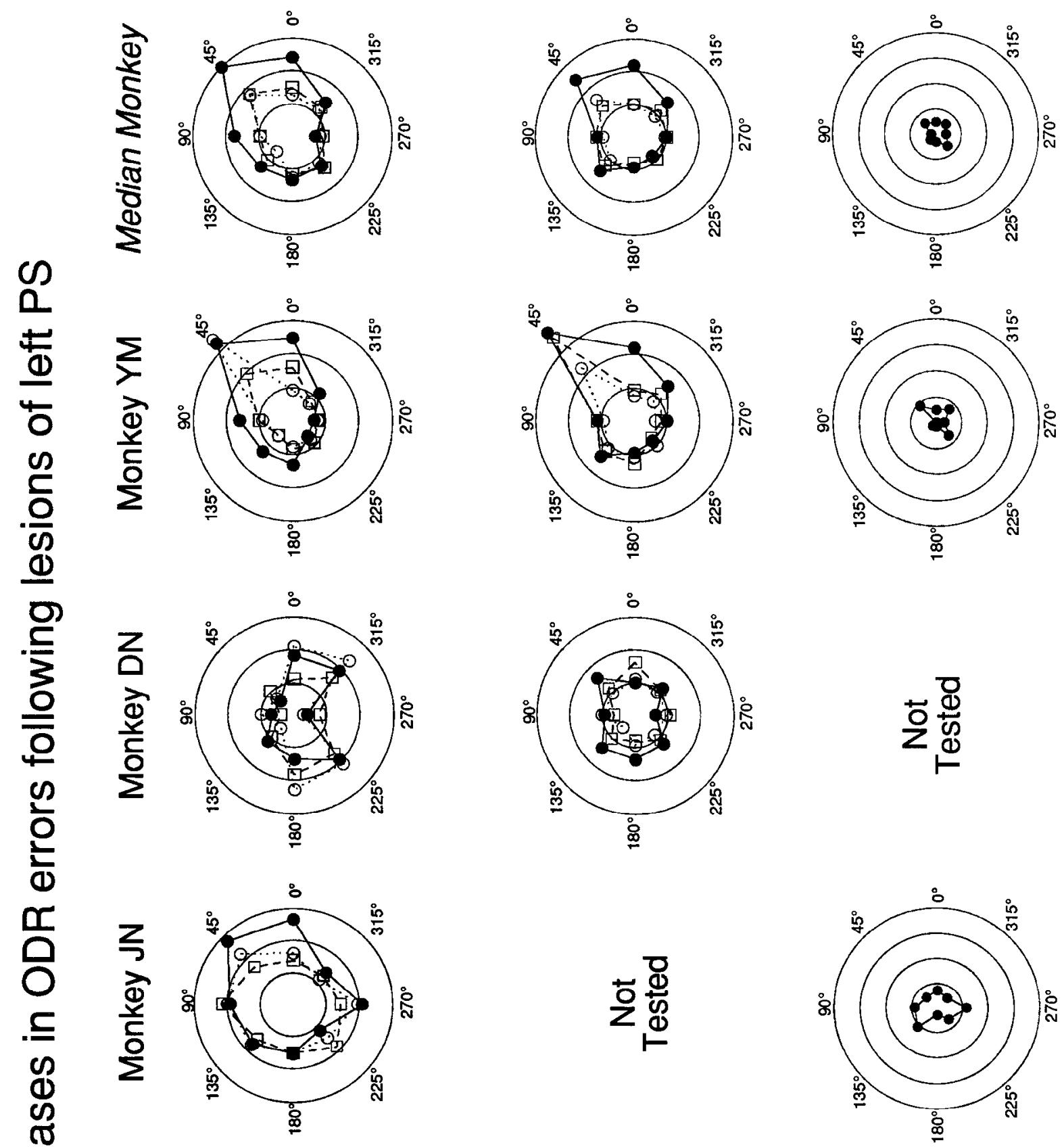

है
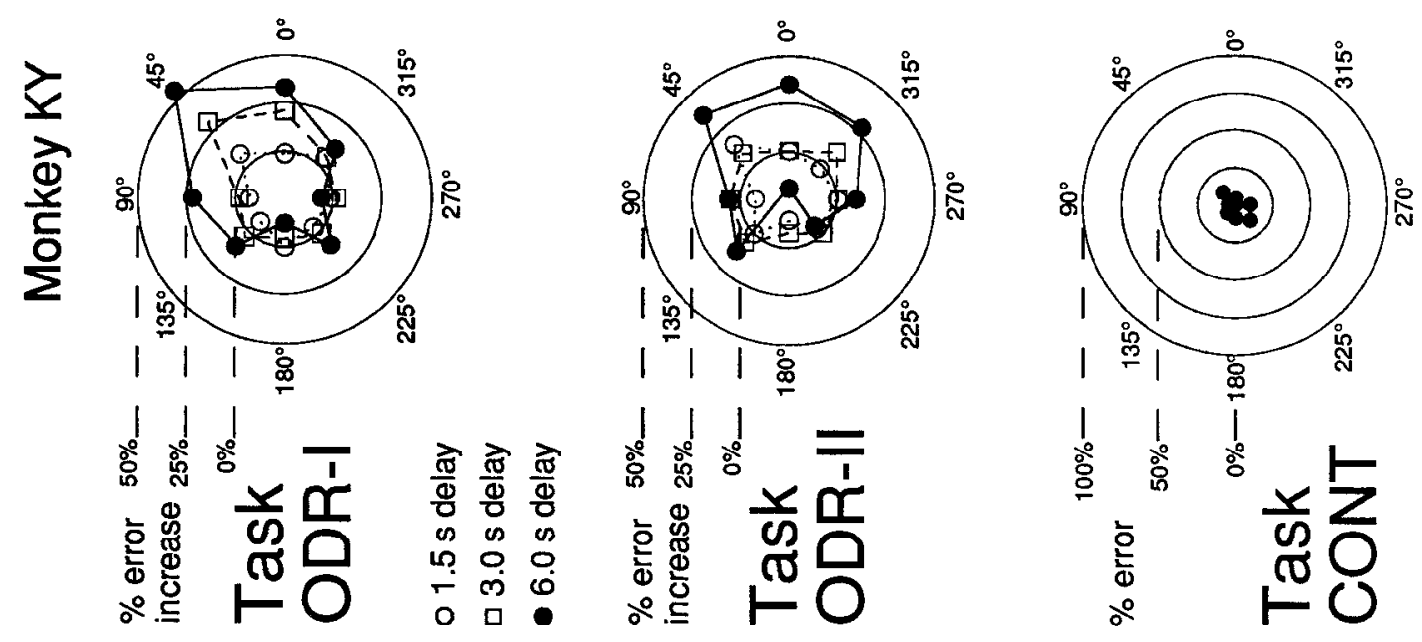

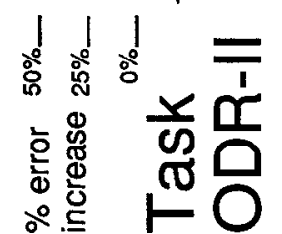


had some difficulty for the leftward direction at $6 \mathrm{sec}$ delays. Damage caused by single-neuron recording in the right prefrontal area (see Fig. 3) may have caused these difficulties; however, this is only speculative as we did not formally test the monkey on these ODR tasks before the recording commenced.

Following the left PS lesion (Fig. 4, right column), monkey KY's errors on ODR tasks substantially increased. Most noticeably, KY now made over $50 \%$ errors for the upper right $\left(45^{\circ}\right)$ cues with 6 sec delay in both ODR-I and ODR-II. There were significant error increases for other directions as well, again mostly for longer delay trials. On the ODR-II task KY now had substantial errors $(>25 \%)$ for all three right field cues when tested with 6 sec delays. There was also some worsening of its preoperative difficulty with upper left field cues.

These increased incidences of errors following the lesion principally reflect decreases in saccade accuracy because monkey KY nearly always made a saccade within the allotted $0.5 \mathrm{sec}$ on virtually every trial, both before and after its lesion (nor were saccadic reaction times affected by the lesion, as shown further below). Therefore, to analyze the deficits further, we compared two-dimensional scatter plots of the end points of the response period saccades made before and after the lesions. Figure 5 shows plots of the end points of saccades made by monkey KY on the ODR-I task following $45^{\circ}$ cues, the direction for which the error scores indicated the most substantial performance decrement following the lesion. For comparison, the figure also shows saccadic end points following the opposite direction cues $\left(225^{\circ}\right.$, down and left), a direction for which the error scores indicate only small decrements in performance. The top row of Figure 5 shows that saccade distributions for these two cue directions were fairly comparable before the lesion, with nearly all saccades ending in the correct quadrant and with a moderate increase in the overall scatter of saccadic end points with lengthening delays for both directions. However, the bottom row of Figure 5 shows that following the left PS lesion there was a general increase in the scatter of saccadic end points for the $45^{\circ}$ cue direction under both the $3 \mathrm{sec}$, and particularly the $6 \mathrm{sec}$ delay condition. In fact, 10 (of 23 ) saccades in the $45 \% \mathrm{sec}$ condition ended outside the upper right quadrant following the lesion, whereas all ended inside it before the lesion. In contrast, the accuracy of saccadic end points for the $225^{\circ}$ cue direction was little affected by the lesion, even with the 6 sec delay.

This pattern of an increased variability of saccadic end points for longer delays following the left PS lesion was found to some extent for all three rightward (contralateral) cue directions $\left(45^{\circ}\right.$, $0^{\circ}, 315^{\circ}$ ), as well as for upward $\left(90^{\circ}\right)$ cues. Figure 6 shows saccadic end points plotted for all eight cue directions at each of

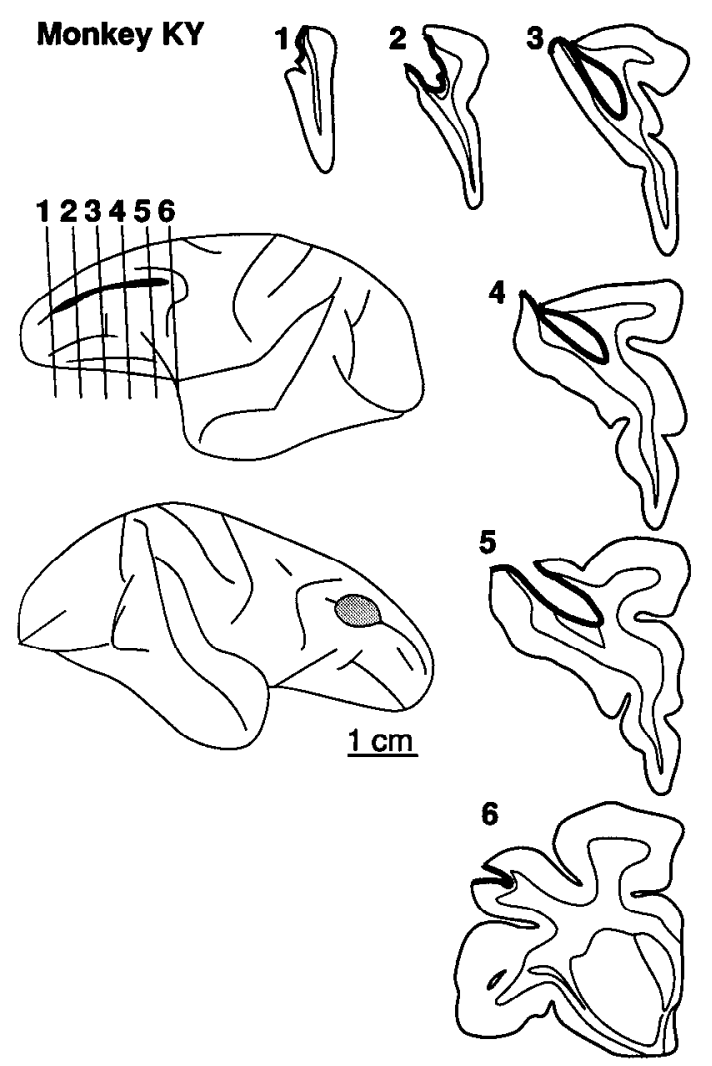

Figure 3. Reconstruction of the lesion in the left hemisphere of monkey $\mathrm{KY}$. The aspirated area is shown on the lateral view of the brain (black zone) and in coronal sections (shaded outline). The lesion extended through the entire PS in the left prefrontal cortex, but the AS was not involved. Single-neuron activity was recorded from the right PS cortex before the lesion experiment; this recording area is indicated by the shaded area in the lateral view of that hemisphere.

the three delays, together with ellipses indicating the horizontal and vertical variability of the end points. With $1.5 \mathrm{sec}$ delays, the monkey usually made similarly accurate saccadic eye movements both before and after the lesion. However, for both the 3 and 6 sec delays, saccades directed to the right visual field cues, that is, to the $45^{\circ}, 0^{\circ}$, and $315^{\circ}$ locations, and to the upper field (the $90^{\circ}$ location) cues, showed greater variability after the lesion than before, and this effect was larger at $6 \mathrm{sec}$ delays than at 3 sec delays. By contrast, the variability of saccades directed to left (ipsilateral) hemifield cues was similar before and after the lesion at all delays.

\footnotetext{
Figure 2. Summary of the effect of the left hemisphere lesion of PS cortex on ODR-I and ODR-II task performance for all four monkeys. The top row shows the increase (after as compared with before the lesions) in the percentage of trials on which the monkeys' saccades terminated outside of the rewarded window (error trials) for the ODR-I task (no targets present at end of the delay period). The middle row shows the same statistic for task ODR-II (all eight targets present at end of the delay period). The bottom row shows performance level on the control I task. Each point plotted in the ODR tasks (I and II) corresponds to the increase in error at one of the eight cue directions, with the three different delay period durations tested at each direction indicated by different symbols as designated in the key. The differcnce statistic plotted represcnts crror pcrcentages on three different testing days conducted approximately 2 weeks following the PS lesions less the scores on the same direction/delay conditions taken from sessions conducted shortly before the lesion was given. Data points within the innermost circle represent improvement following the lesion. Only absolute levels of performance after the lesions are plotted for the control task, as percentage increase in error for this task was negligible. Monkey $J N$ did not learn ODR-II. Monkey $D N$ used delays of 1, 2, and $4 \mathrm{sec}$, and the $1 \mathrm{sec}$ condition served as a control for sensorimotor loss in this animal. The rightmost plots show the medians for each of the 24 direction/delay combinations across the four monkeys. Notice that for both ODR tasks all three directions $\left(45^{\circ}, 0^{\circ}\right.$, and $\left.315^{\circ}\right)$ contralateral to the lesions have a clear increase in errors for 6 sec delays, with $45^{\circ}$ and $0^{\circ}$ having a greater than $25 \%$ increase.
} 

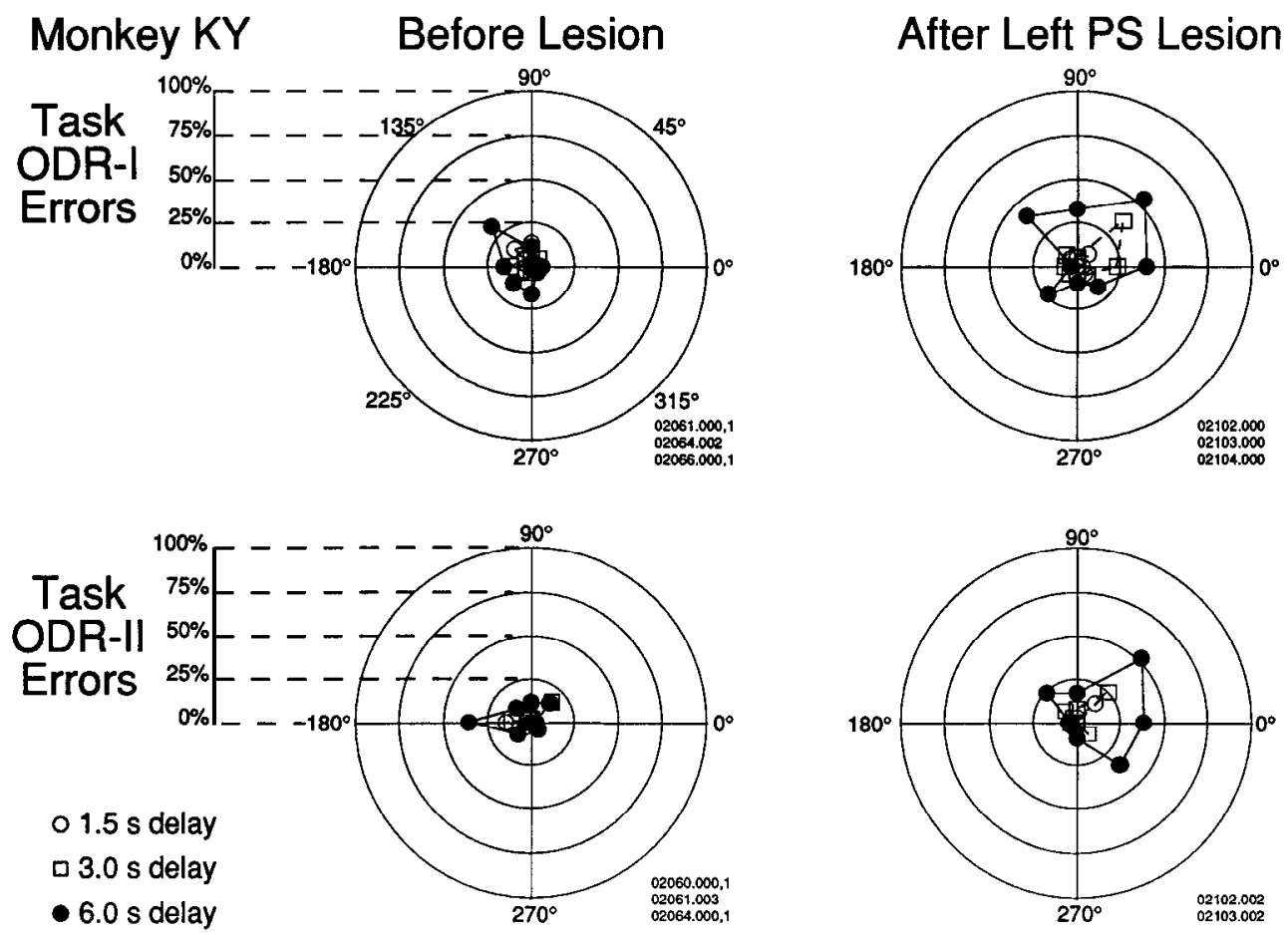

Figure 4. Summaries of ODR-I and ODR-II task performance of monkey KY before and after its left PS lesion. The radial plots show the percentages of trials on which the monkey's saccade terminated outside the rewarded window, constituting an error trial. Each point plotted corresponds to the error measure at one of the eight cue directions, with the three different delay periods (1.5, 3 , and $6 \mathrm{sec})$ tested at each direction indicated by different symbols as designated in the key. The left column averages across 3 consecutive testing days shortly before the lesion was given, and the right column averages days 8,9 , and 10 following the lesion of PS cortex in the left hemisphere. Notice that for directions contralateral to the hemisphere receiving the lesion, the percentage of errors in the $6 \mathrm{sec}$ delay condition substantially increased in both the ODR-I and ODRII tasks. In contrast, errors for most other directions and errors at shorter delays did not increase or increased only modestly. A relatively larger error for $135^{\circ}$ and $180^{\circ}$ cues was evident before the lesion, and may reflect the slight damage in the PS of the right hemisphere (see Fig. 3) caused by recording there before designating this monkey for the present study. Following the aspiration lesion of the left PS (right column), this problem with the $135^{\circ} \mathrm{sec}$ location worsened somewhat; however, $180^{\circ}$ performance improved.

Monkey JN. As shown in Figure 7, JN's first lesion was located in the left hemisphere and included the posterior two-thirds of PS as well as a large portion of the adjaccnt cortcx of the supcrior dorsolateral convexity (DLC). The AS was spared. About 2.5 months after the first lesion, a comparable lesion was made in the right hemisphere, the AS again being spared. This monkey's lesions were, therefore, reasonably symmetrical bilaterally.

Figure 8 shows both the percentages of error trials (top row) and the average size of saccade errors (bottom row) for monkey JN. Only ODR-I is shown because JN was not tested on the ODR-II task. Preoperatively, ODR-I performance was excellent for all directions and delays. After the large left PS +DLC lesion, the most marked increase in error size and percentage was for the cue locations in the right visual field (contralateral to the lesion), especially for cue directions $45^{\circ}$ and $0^{\circ}$ in conjunction with the longest delay condition. A considerable performance decrement was also observed for the cue locations in the lower visual field $\left(270^{\circ}\right)$ for $6 \mathrm{sec}$ delays, and a milder decrement in a few of the left visual field conditions. The second-stage right prefrontal lesion produced only minor additional disruption of the monkey's ODR-I performance.

Like monkey KY above, JN consistently made saccades within the allotted $0.5 \mathrm{sec}$ for nearly all trials in all directions and delays, both before and after its lesions. Therefore, again the deficits following the lesions reflected inaccurate saccades. For this monkey we chose the left $\left(180^{\circ}\right)$ and right $\left(0^{\circ}\right)$ cue directions for showing the effects of the lesions on saccadic end points.
Figure 9 shows that saccadic end point distributions for 3 and $6 \mathrm{sec}$ delays became considerably more scattered for the right $\left(0^{\circ}\right)$ cue direction following the left hemisphere lesions. However, there was little effect on the leftward $\left(180^{\circ}\right)$ trials at any of the delay times. Following the addition of the right hemisphere lesion (Fig. 9, bottom row), a large increase in saccade scatter for leftward cues is apparent for the 1 and 4 sec delays; however, the performance for the rightward cues improved, almost to preoperative levels.

Figure 10 shows monkey JN's saccadic end point distributions and variability ellipses across all cue directions, delay times, and lesion conditions. This plot emphasizes the primarily contralateral nature of the scatter increases following the left PS +DLC lesions, and the milder effects of the second, right hemisphere lesion on variability in the left field. Again, notice the recovery in the ipsilateral field following the second lesion.

Monkey DN. DN's lesion was made in the anterior one-half of PS in the left hemisphere (Fig. 11). The lesion did not encroach on the caudal half of PS, and no damagc was observed in AS. The right prefrontal cortex was used for single-neuron recording before the ablation, and cortical damage was observed in both the white and gray matter in the middle portion of PS, presumably caused by numerous penetrations of recording electrodes.

The contralateral behavioral deficits seen in previous monkeys were again observed in this monkey for the ODR-I task. Its right prefrontal cortex had been severely damaged during 

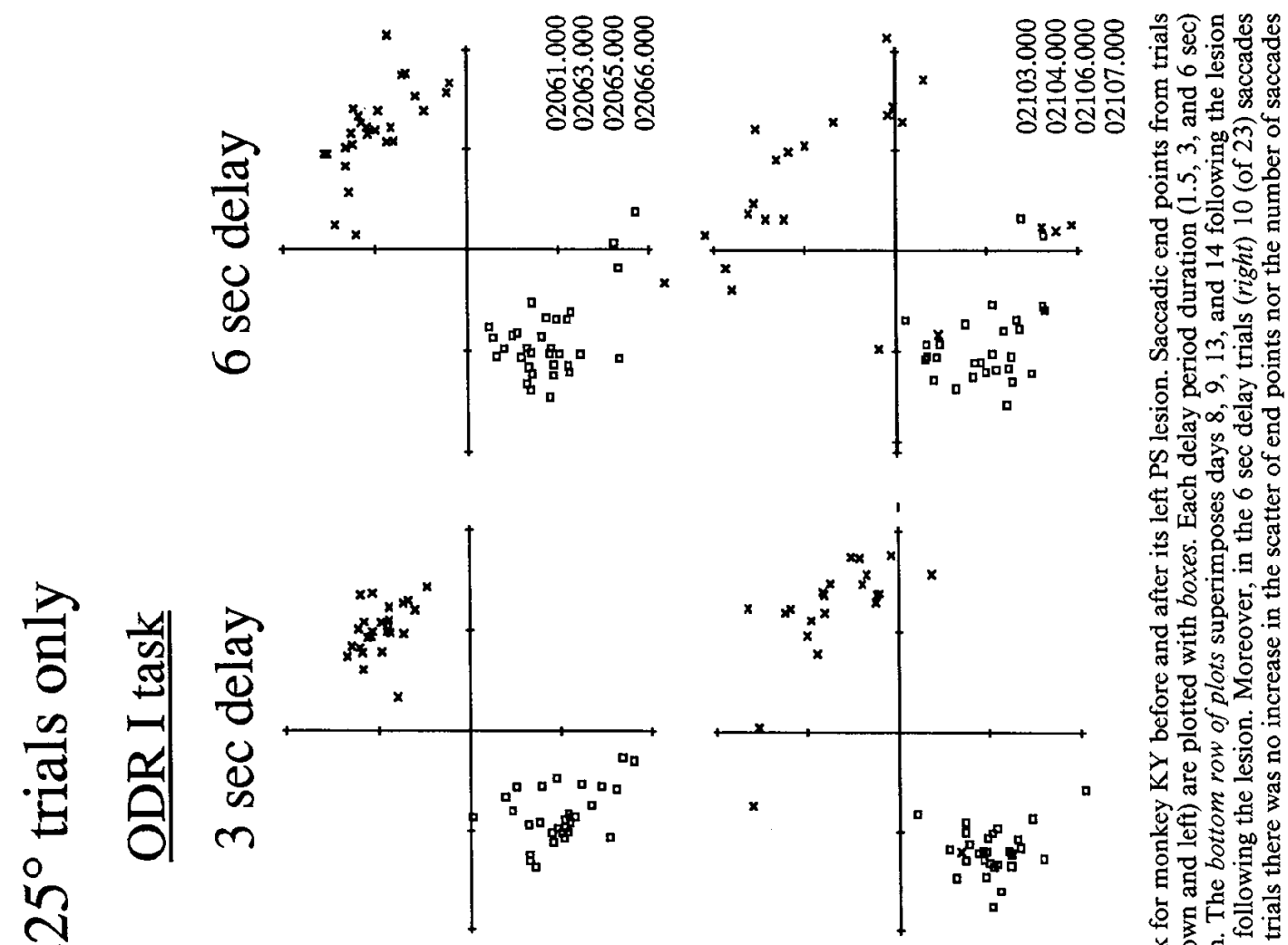

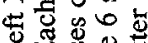

岄总焉

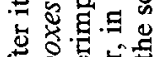

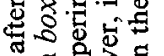

प्दु के

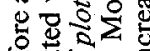

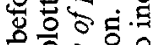

西总递

空运

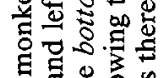

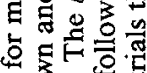

กิ
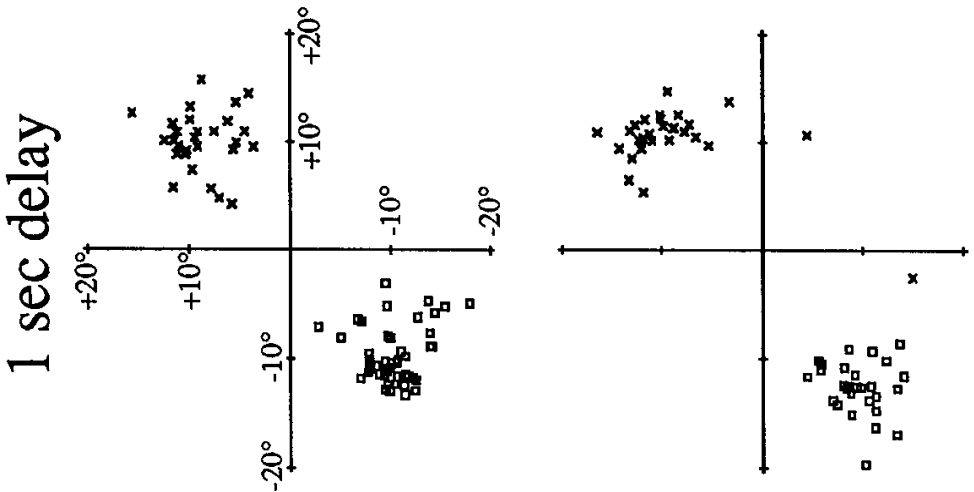

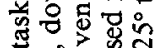

สำ

if

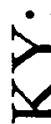

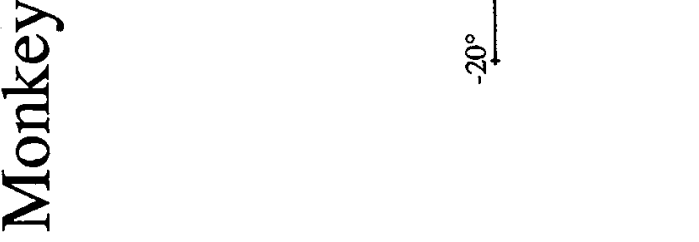

Ðั)
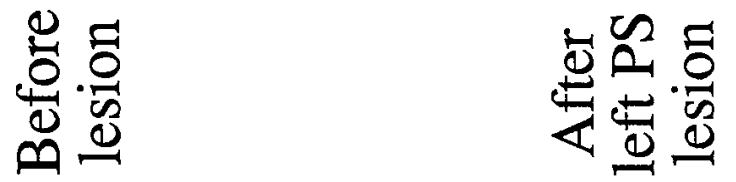

ํำ

О등

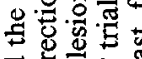

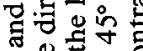

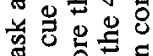

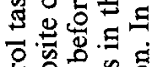

영융릉ㅎㅇ

岁至它

式可邧。

此

要索芛

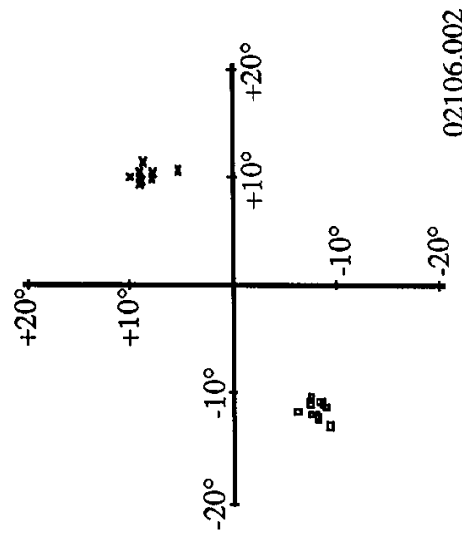

궁

氞总总

in कि

$8 \times$

들 茟

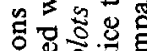

븡응

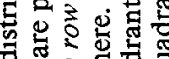

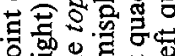

员医果

要焉象宫

을열영

용용

它

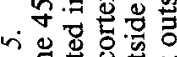

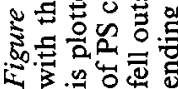



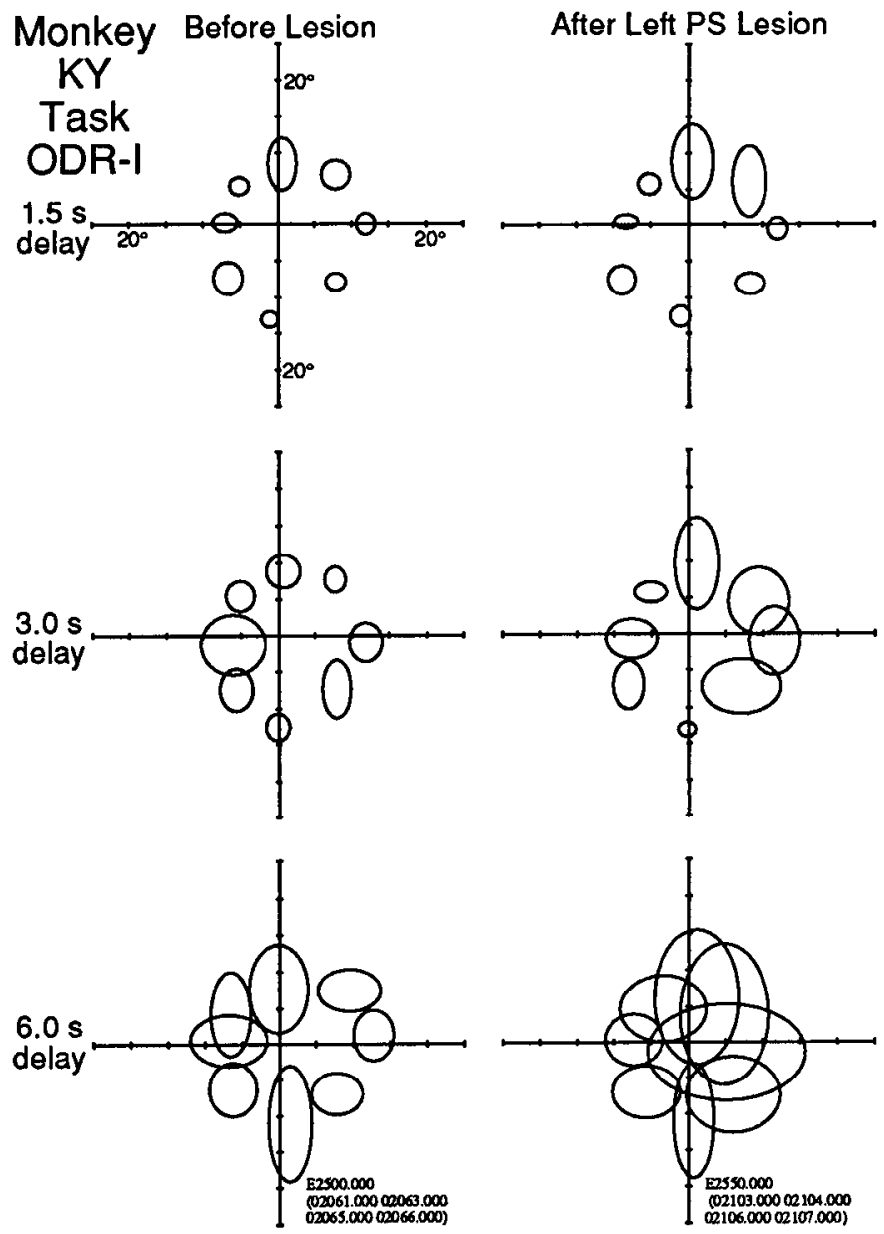

Figure 6. Saccadic end point variance of monkey KY for all cue directions in the ODR-I task before and after its left PS lesion. The three delay periods tested $(1.5,3$, and $6 \mathrm{sec})$ are plotted separately in the three rows. The eight ellipses in each plot convey overall saccade accuracy for each cue position. Each ellipse is centered at the mean $\mathrm{x}$ - and $\mathrm{y}$-coordinates of the saccadic end points for its cue, and the horizontal and vertical axes of the ellipses equal the SD of these $\mathrm{x}$ - and $\mathrm{y}$-coordinates, respectively. The left column of plots combines four sessions conducted just before the lesion was made. The right column combines sessions conducted $8,9,13$, and 14 days following the lesion of PS cortex in the left hemisphere. Notice that in the $6 \mathrm{sec}$ delay condition all three rightward directions, and the upward direction, have substantial increases in ellipse size following the left hemisphere PS lesion. This increase is more modest for the shorter $(1.5$ and $3 \mathrm{sec})$ delays and absent for the other four directions at any delay.

single-neuron recording, and this damage was associated with a large behavioral deficit in the ODR-I task for visual cues presented in the lower left quadrant of the visual field (Fig. 12, top). However, no such clear behavioral deficits were observed in the ODR-II task, even in the longest delay condition (Fig. 12 , bottom). After the subsequent PS lesion was made in the left hemisphere, additional behavioral decrement was observed in the ODR-I task when visual cues were presented in the lower right quadrant of the visual field, but again without concomitant deficits in the ODR-II task.

Unlike the other three monkeys, the lower scores of DN, primarily on trials directed toward the lower visual field in the ODR-I task, not only reflected inaccurate saccades, but also an absence of eye movements within the allotted 0.5 sec on many trials. Instead, this monkey often maintained central fixation

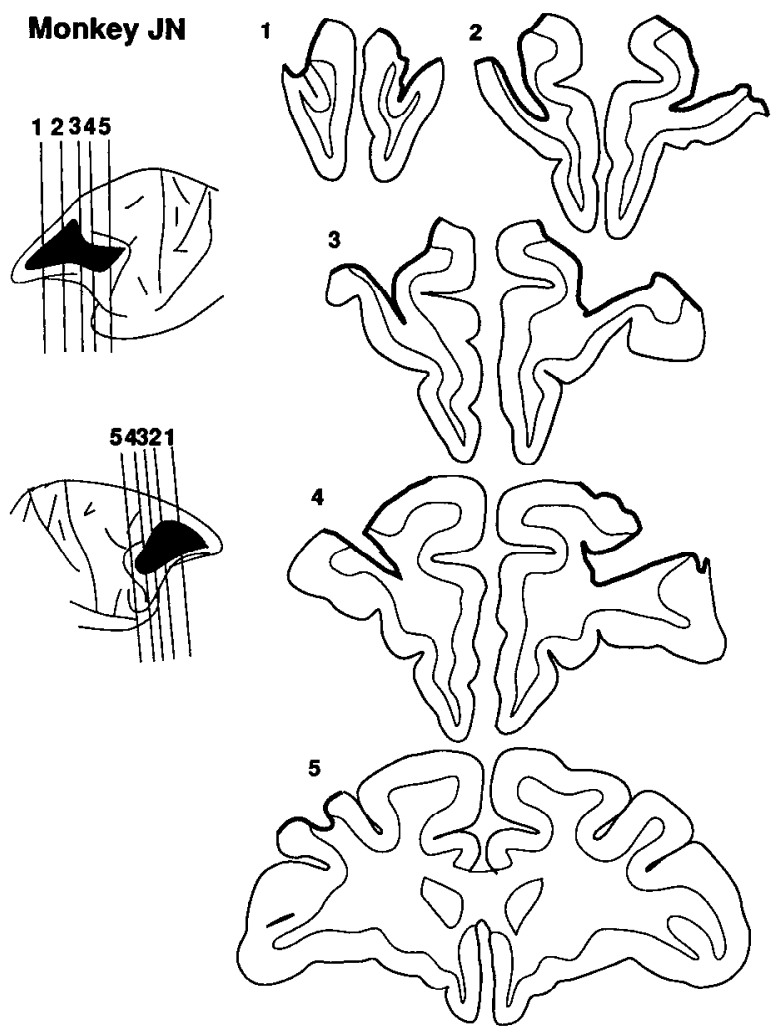

Figure 7. Reconstruction of the lesions of monkey JN. Both lesions included the PS plus a large portion of the DLC of prefrontal cortex, sparing only the dorsomedial and ventrolateral margins of the convexity. Although both lesions extended to the posterior end of the PS, the AS was spared (section 5).

long after the fixation target disappeared. This behavior occurred for nearly $45 \%$ of the $225^{\circ}$ trials and $40 \%$ of the $270^{\circ}$ trials in every test session before and after the lesions. We avoided this monkey's problematic lower field by making saccadic end point plots for horizontal cue locations as shown in Figure 13. However, these plots primarily indicate that monkey DN also had poor saccade metrics in both horizontal directions before its aspiration lesion, and it is hard to discern additional scatter in saccadic end points for these directions following its lesion. Figure 14 shows saccadic end point plots and variability ellipses of monkey DN for ODR-I performance. These plots indicate that the experimental lesion caused a moderate deficit for the combination of $4 \mathrm{sec}$ delays and the three cue directions $\left(90^{\circ}, 45^{\circ}, 0^{\circ}\right)$ in the upper right quadrant.

Monkey YM. For monkey YM (Fig. 15), the first-stage lesion was located in the posterior part of the left prefrontal area. The lesion included the posterior one-third of PS and also part of the anterior bank of the AS, where the FEF is located. About 1.5 months after the first lesion, a second-stage lesion was made in the middle one-third of the right PS; the AS was not included in this second lesion. Thus, this monkey had a caudal PS+AS lesion in the left hemisphere and a middle PS lesion in the right.

Monkey YM performed the ODR task very well (Fig. 16, left column) before receiving its lesions. Following the left hemisphere lesion YM showed a severe deficit for cues presented in the upper right quadrant of the visual field in the ODR-I task (Fig. 16, middle column), and also in the ODR-II task (not shown). However, unlike monkeys KY and JN, this deficit was 

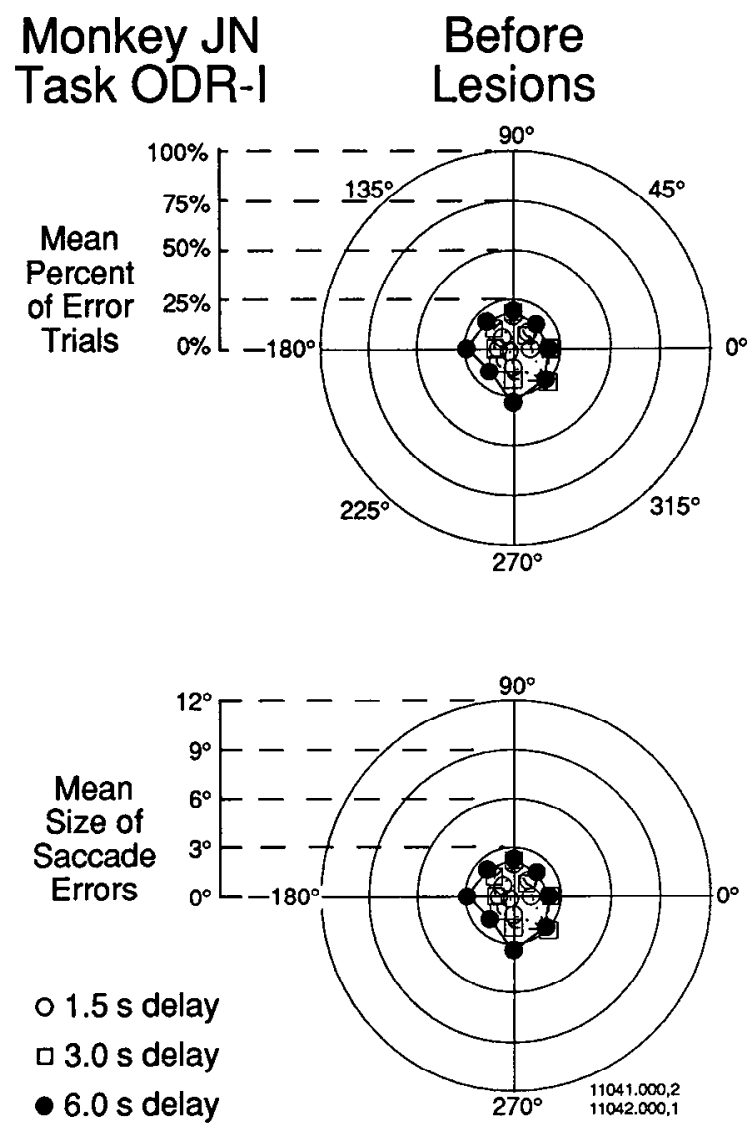
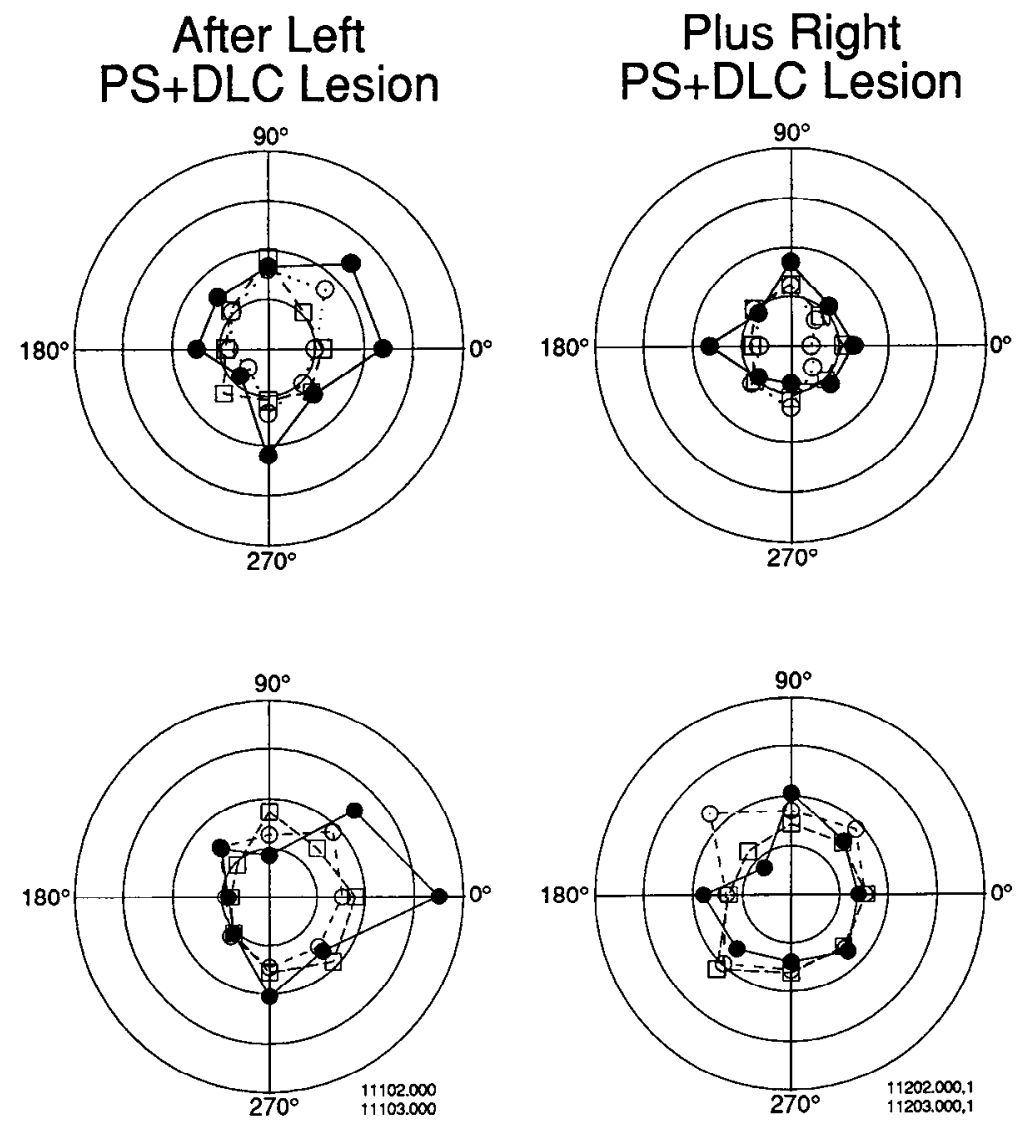

Figure 8. Summaries of ODR-I task performance of monkey JN before and after each of its lesions. The top row shows the percentages of trials on which the monkey's saccade fell outside the rewarded window, constituting an error trial. The bottom row shows the average size of the saccade error, that is, the distance (in degrees subtended) between the location of the cue and the end point of the saccade made toward the cue's remembered location. The left column shows both types of performance graphs, averaged across 4 test days and collected just before receiving its first lesion. The middle column shows performance on days 9 and 11 following the lesion in the left hemisphere. The right column shows performance on days 8 and 9 following the subsequent lesion in the right hemisphere. Conventions are as in Figure 4 . Note that both percentage errors and error magnitude increased principally in the upper right $\left(45^{\circ}\right)$, rightward $\left(0^{\circ}\right)$, and downward $\left(270^{\circ}\right)$ directions following the left hemisphere lesion, and also that this increase was principally for the $6 \mathrm{sec}$ delay trials. Following the right hemisphere lesion, these deficits appreciably lessened.

present even in the $1.5 \mathrm{sec}$ delay condition, and it was still present 1.5 months later, when the second ablation was performed.

After the second lesion in the right hemisphere, monkey YM exhibited difficulty in performing saccades to the left visual field for the first time, and this deficit was expressed in both ODR tasks. The most pronounced impairment was for $180^{\circ}$ and $225^{\circ}$ trials with $6 \mathrm{sec}$ delays in the ODR-I task. In contrast, performance was nearly perfect on the $135^{\circ}$ and $270^{\circ}$ trials under all delay conditions.

Unlike monkey $\mathrm{JN}$, the addition of the right hemisphere lesion in YM did not relieve its deficits in the right hemifield caused by the first lesion. Instead, YM's deficit at $0^{\circ}$ was exacerbated in the ODR-I task, and its severe deficit at $45^{\circ}$ was not ameliorated. In addition, the lower right quadrant was affected for the first time, particularly at the $315^{\circ}$ location, in both ODR tasks, and at the shorter as well as longer delay periods.

Saccadic end point distributions for cues in the $45^{\circ}$ and $225^{\circ}$ directions for monkey YM are shown in Figure 17. There was a large increase in the scatter of saccadic end points for the $45^{\circ}$ (upper/contralateral) cues following the left hemisphere lesion, which was most dramatic for the 3 and $6 \mathrm{sec}$ delay times. In contrast, saccades to the $225^{\circ}$ cues were hardly affected at any delay. Following the subsequent middle PS lesion in the right hemisphere, there was a mild increase in the variability of saccadic end points for the $225 \% \mathrm{sec}$ condition and little change for the $45^{\circ}$ trials.

Figure 18 shows monkey YM's saccadic end points and variability ellipses for all conditions. The primary effect of the left hemisphere is in the right field, especially the upper quadrant, whereas a milder effect of the subsequent right PS lesion on saccade scatter is apparent only for the lower left field cues $\left(225^{\circ}\right)$ at the 6 sec delay.

\section{Parameters of oculomotor response}

To understand better the nature of the monkey's deficits in the ODR tasks, we used the event buffer files to calculate saccadic reaction time (latency) and average saccadic velocity for each saccade made in the response period of the ODR tasks. In addition, using analog buffer files, we constructed trajectories of these saccades.

Reaction time. Figure 19 shows saccadic reaction times in the ODR-I task for all four monkeys, before and after their lesions. Saccadic reaction time was defined as the time interval between the disappearance of the fixation target and the initiation of the saccade. As long as the lesions did not include the AS, where 
Figure 9. Saccadic end point distributions in the $0^{\circ}$ and $180^{\circ}$ trials of the ODR-I task for monkey JN, preoperatively and following each of its unilateral PS + DLC lesions. Saccadic end points from trials with the $0^{\circ}$ cue (right) are plotted with $\times$ 's, whereas trials with the opposite cue direction $\left(180^{\circ}\right.$, left $)$ are plotted with boxes. Data for top row of plots combine 2 consecutive test days shortly before the first lesion was made. Data for the middle row plots combine days 9 and 11 following the lesion of PS+DLC cortex in the left hemisphere. The bottom row of plots combines days 8 and 9 following the subsequent lesion of PS+DLC cortex in the right hemisphere. Notice that in the rightward $(X)$ trials that the scatter of saccadic end points following the left hemisphere lesion (middle row) increased substantially relative to the preoperative data (top row) especially for the 3 and $6 \mathrm{sec}$ delay trials, whereas for the leftward trials (boxes) there was no increase in the scatter of end-points for any of the delays. Note also the subsequent disruption of saccadic end points for leftward trials of all delays following the subsequent right hemisphere lesion (bottom row), with an accompanying improvement in the saccadic end points for leftward trials.

Figure 10. Saccadic end point variance of monkey $\mathrm{JN}$ for all cue directions in the ODR-I task before its lesions and after each lesion. The three delay period durations $(1.5,3$, and $6 \mathrm{sec})$ are plotted separately in the three rows. The eight ellipses in each plot convey overall saccade accuracy for each cue position. Each ellipse is centered at the mean $x$ - and $y$-coordinates of the saccadic end points for its cue, and the horizontal and vertical axes of the ellipses equal the SD of these $x$ - and $y$-coordinates, respectively. The left column of plots shows data collected just before the first lesion was given. The middle column of plots shows data from $9 \mathrm{~d}$ following the lesion of PS cortex in the left hemisphere. The right column of plots shows data from $8 \mathrm{~d}$ following the subsequent lesion of PS cortex in the right hemisphere.
Monkey JN Task ODR-I: $0^{\circ}$ and $180^{\circ}$ trials only
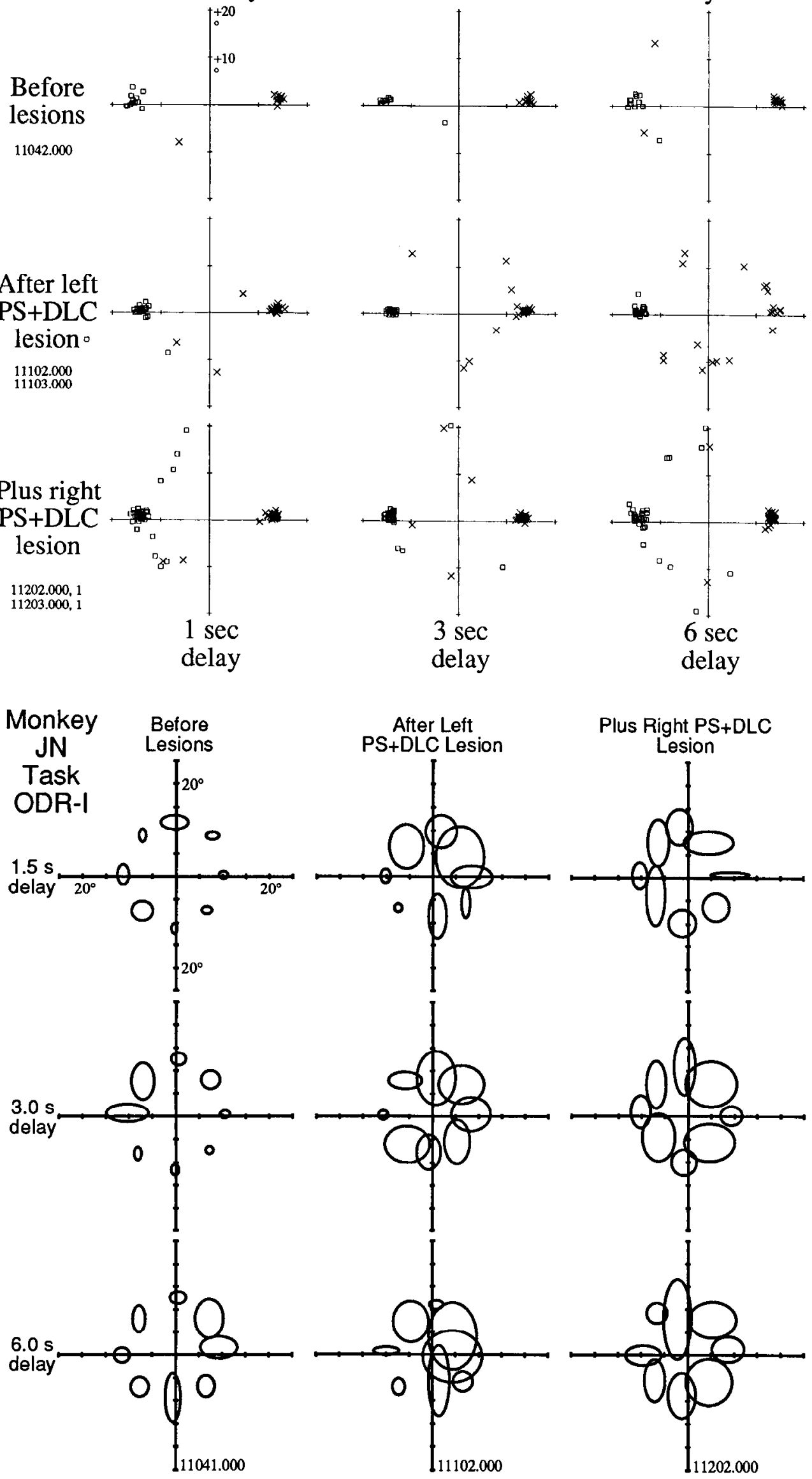

Plus Right PS+DLC
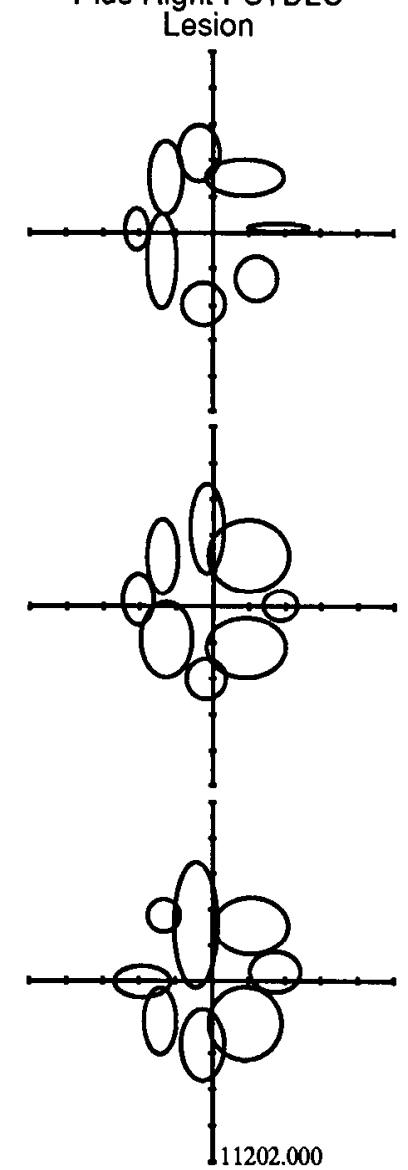


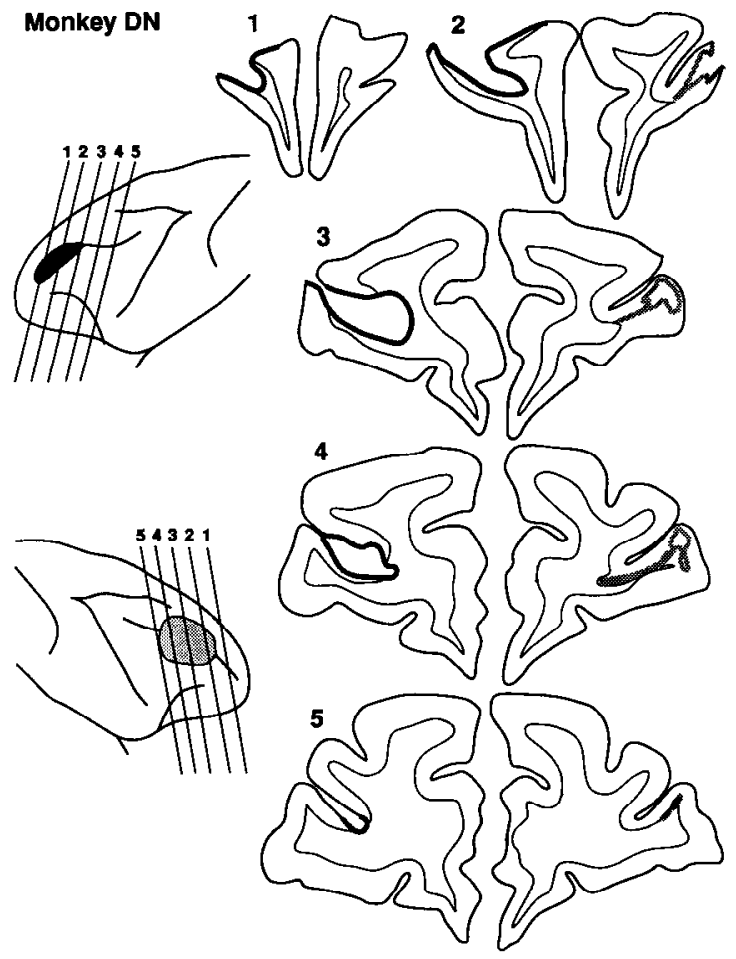

Figure 11. Reconstruction of the lesion in the left hemisphere of monkey DN. The lesion involved the anterior half of the PS area in the left prefrontal cortex. The AS and FEFs were not involved. Single-neuron activity was recorded from the right prefrontal cortex and the numerous electrode penetrations caused damage in the right prefrontal cortex as indicated by the shaded area. the FEF is located, there were no marked changes in reaction times either to ipsilateral or contralateral cues (see monkeys $\mathrm{KY}, \mathrm{DN}$, and JN). Further, these values were relatively consistent among different delay conditions for each monkey. Thus, unlike the behavioral scores, there were no significant changes in saccadic reaction times in the ODR-I task after the unilateral prefrontal lesions. Likewise, the lesions sparing FEF caused no significant changes in saccadic reaction times for the ODR-II task (not shown).

However, including the FEF in the lesion significantly lengthened reaction times. After monkey YM received a lesion of the left prefrontal cortex that included FEF, reaction times in both ODR tasks were markedly longer for saccadic eye movements directed to the upper right visual field $[x=250 \mathrm{msec}$ (all delays) for right upper field; $x=200 \mathrm{msec}$ and $220 \mathrm{msec}$ for $270^{\circ}$ and $180^{\circ}$ targets, respectively; $F(3,192)=46.24, p<0.0001$ for all comparisons involving right upper field]. These differences remained after the second-stage lesion in the right hemisphere. However, there was no increase of reaction time for saccadic eye movements toward the left visual field after this right prefrontal lesion that did not include $\operatorname{FEF}[F(91,284)=0.044 ; p$ $>0.05]$.

Saccadic velocity. The average velocity of each saccade was calculated from saccade duration and amplitude stored in the event buffer files. Figure 20 shows plots of saccadic velocity for task ODR-I for all monkeys, both before and after their lesions. As in the case of reaction times, no marked differences were observed in velocities for any eye movement directions when the lesion spared the FEF (see monkeys KY, DN, and JN). Although the mean values differed among directions of saccadic eye movements, these values were very consistent across the
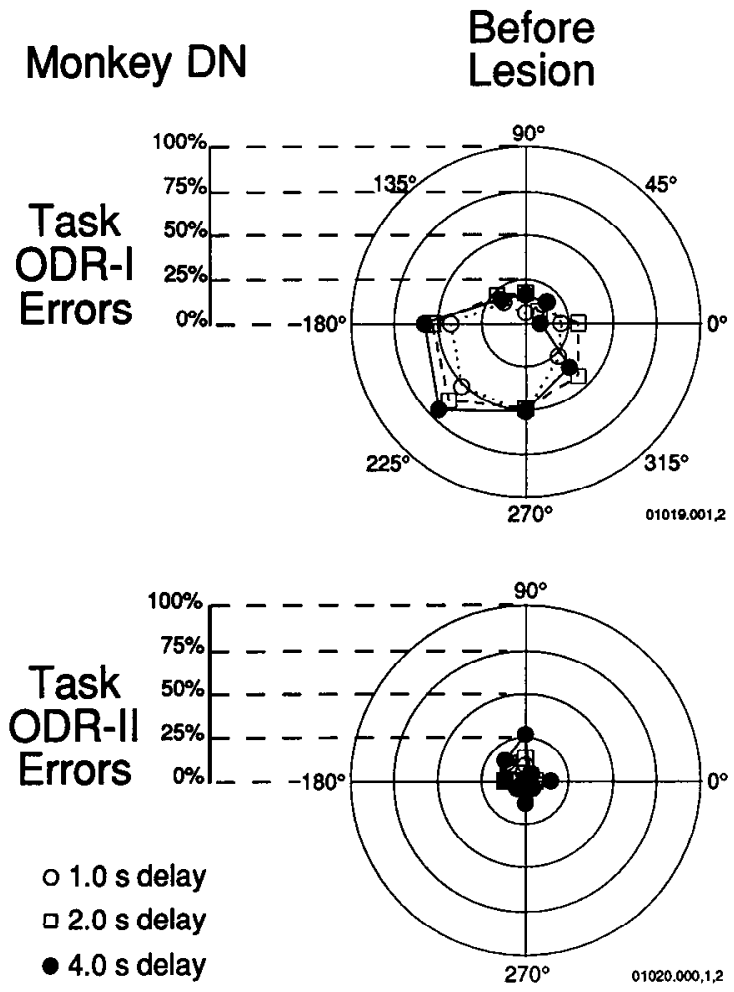

After Left Anterior PS Lesion
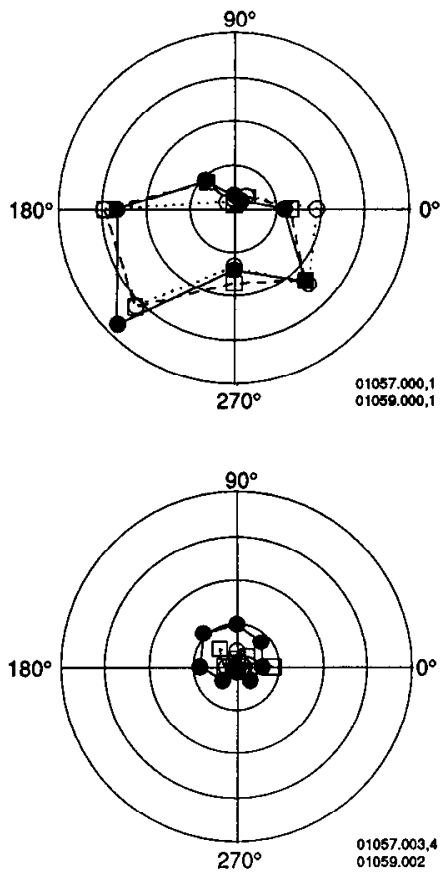

Figure 12. Summaries of ODR-I task and ODR-II performance of monkey DN before and after its left PS lesion. The left column shows both types of performance graphs, averaged across 4 test days and collected just before receiving its lesion. The right column shows performance for days 12 and 14 following the lesion of anterior PS cortex in the left hemisphere. Conventions are as in Figure 4. The poor scores for the left/down directions and neighboring directions $\left(270^{\circ}\right.$ and $\left.180^{\circ}\right)$ both before and after the left hemisphere lesion may reflect the damage to the PS cortex caused by recording from the right hemisphere before the monkey was assigned to this behavioral experiment; however, no comparable data were obtained before the recording began. 

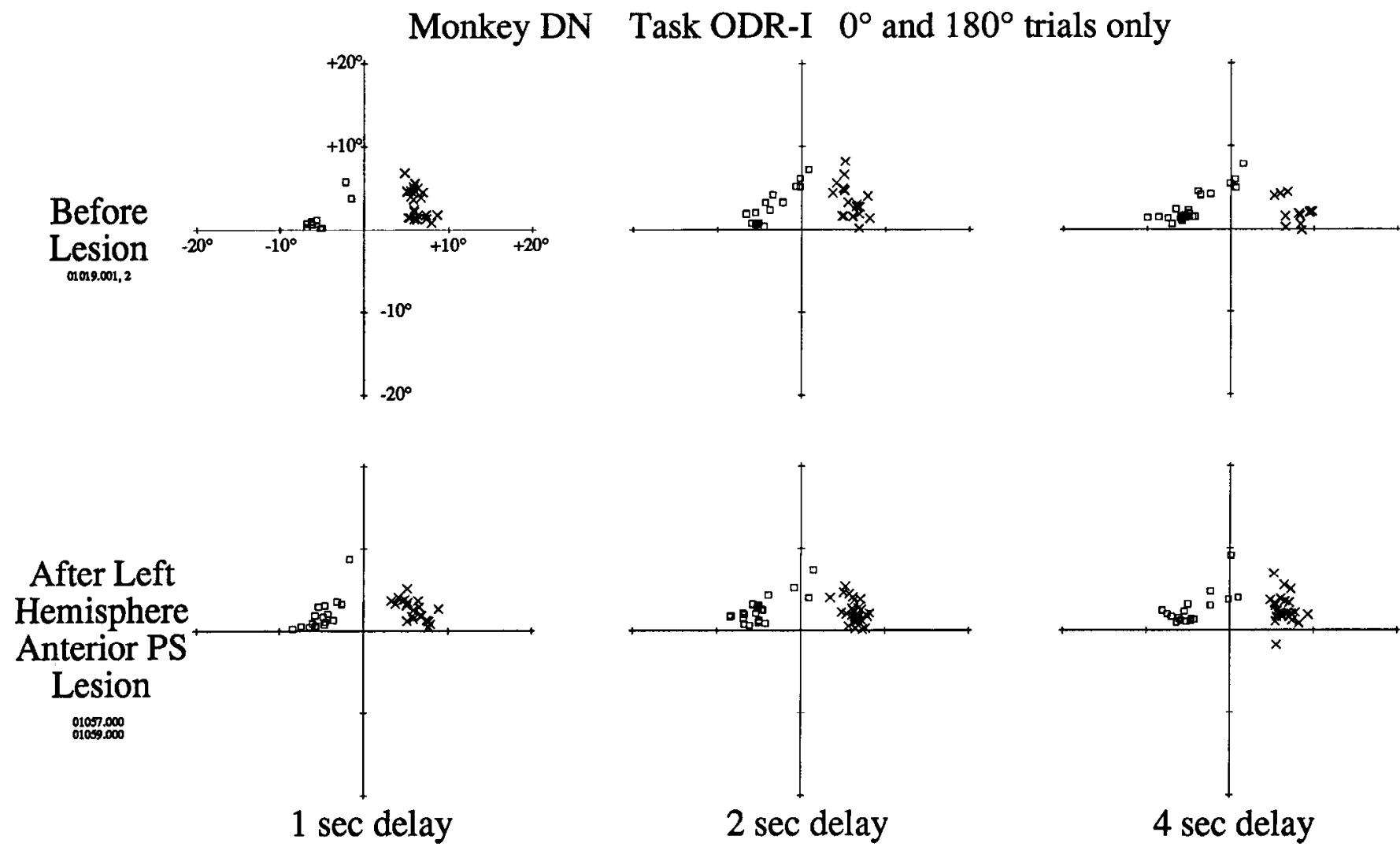

Figure 13. Saccadic end point distributions in the $0^{\circ}$ and $180^{\circ}$ trials for monkey DN on task ODR-I before and after its left PS lesion. Saccadic end points from trials with the cue having a $0^{\circ}$ (rightward) direction are plotted with $\times$ 's, whereas trials with the opposite cue direction (180, left) arc plottcd with boxes. Data for top row of plots were collected $4 \mathrm{~d}$ before the lesion. The bottom row of plots are from 12 and $14 \mathrm{~d}$ following the lesion of anterior PS cortex in the left hemisphere. The poor targeting for the leftward saccades both before and after the left hemisphere lesion may reflect the damage to the PS cortex caused by recording from the right hemisphere before the monkey was assigned to this behavioral experiment; however, no comparable ODR data were obtained before the recording commenced.

different delay conditions within a direction. Thus, unlike the behavioral scores, there were no significant changes in average saccadic velocity in the ODR-I task after the unilatcral prefrontal lesions. Likewise, the lesions sparing FEF caused no significant changes in average saccadic velocity for the ODR-II task (not shown).

However, when the FEF was included in the lesion, we observed a marked decrease in the average velocity of saccades directed to the visual field contralateral to the lesion $[F(1 / 262)$ $=121 ; p<0.0001]$, particularly in the ODR-I task.

Saccadic trajectories. Using analog buffer files, we examined trajectories of saccades. There was no difference in trajectories among different lesion conditions, among different delay conditions, or between ODR and control tasks.

\section{Discussion}

The present study provides new information on the role played by PS and surrounding cortex in spatial delayed-response tasks. First, our findings provide evidence that unilateral prefrontal lesions are sufficient to produce behavioral impairment, though these impairments are not necessarily permanent or profound. Second, the finding that lesions of the left hemisphere affect memory-guided responses to targets in the right visual field and right hemisphere lesions affect performance for the left contralateral hemifield suggests that the organization of the spatial memory system is lateralized and crossed, similar to the organization of the visual system. Third, that memory-guided re- sponses to targets were selectively impaired in a delay-dependent manner while sensory-guided responses to the same dircctions are totally sparcd supports the concept of a mnemonic scotoma, a loss in memory without compromising ability to see the targets or make the appropriate saccades to them. Finally, this is the first demonstration that the deficit caused by lesions in the anterior prefrontal cortex adjacent to the FEF and including the caudal portion of the PS extends to oculomotor as well as classical manual paradigms. Detailed discussion of these points follows.

\section{The PS and mnemonic function}

Among the four monkeys studied in this research, three (KY, $\mathrm{YM}$, and DN) received lesions restricted to the PS and one (JN) received a large lesion in the dorsolateral prefrontal area including the PS. Although the extent and areal location of each lesion differed among the monkeys, the PS area was a common cortical area included in the lesion sites of all monkeys and all monkeys exhibited basically the same pattern of behavioral deficits in the ODR tasks. The involvement of this cortex in spatial mnemonic functions has been confirmed repeatedly by a variety of techniques, including cortical ablation, cryogenic depression, single-unit recording, and psychopharmacological studies [see reviews by Fuster (1989) or Goldman-Rakic (1987) for detail]. However, the precise nature of the deficit has been subject to different interpretations. The fixation requirement of the ODR used in the present experiments, as well as the use of perimetry 

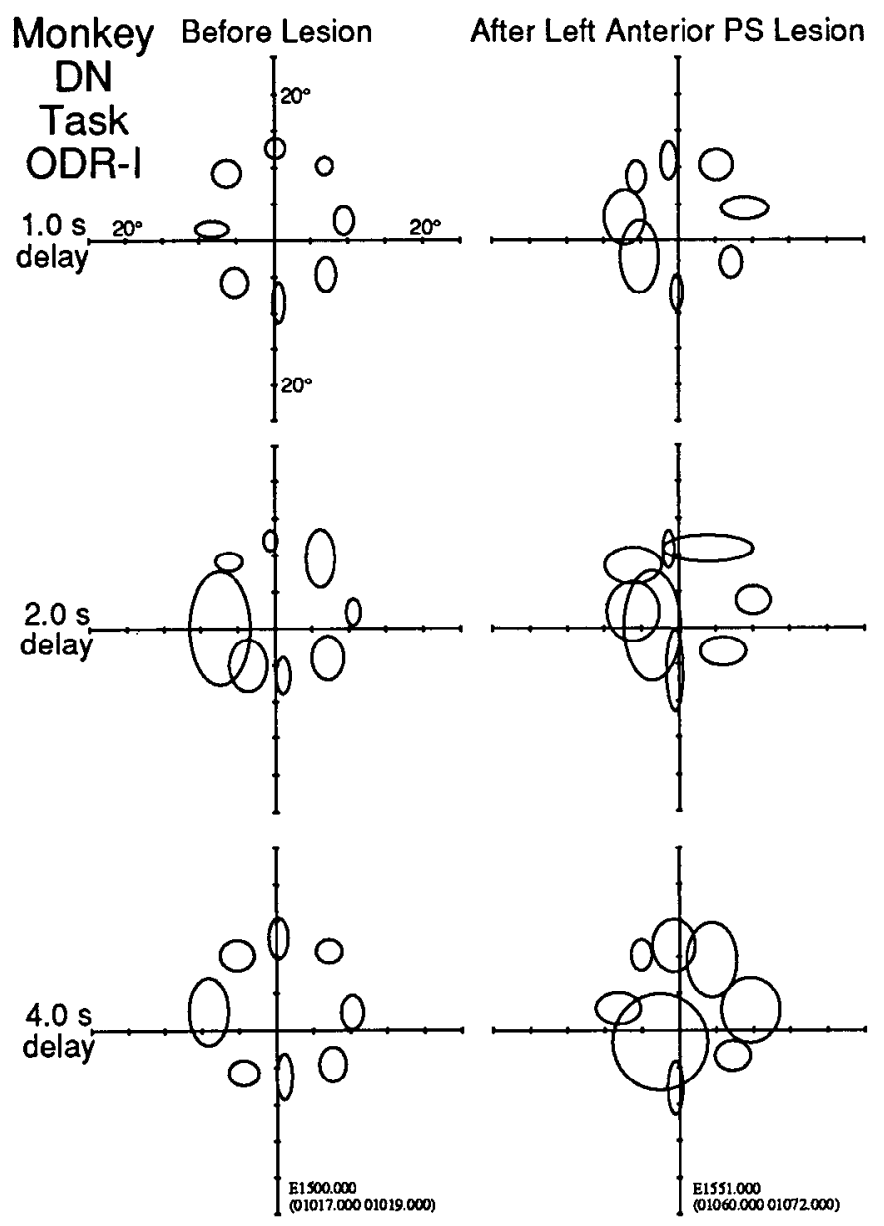

Figure 14. Saccadic end point distributions of monkey DN for all cue directions on task ODR-I before and after its left hemisphere lesion of anterior PS cortex. The three delay periods tested (1, 2, and $4 \mathrm{sec})$ are plotted separately in the three rows. The eight cue positions (eccentricity $1^{\circ}$ ) are plotted with a small $\times$ in each of the six plots, and the eight ellipses in each plot convey overall saccade accuracy for each cue position. Each ellipse is centered at the mean $x$ - and $y$-coordinates of the saccadic end points for its cue, and the horizontal and vertical axes of the ellipscs cqual the SD of these $\mathrm{x}$ - and $\mathrm{y}$-coordinates, respectively. The left column of plots shows data from $2 \mathrm{~d}$ just before the first lesion was made. The right column of plots combines data from 15 and $32 \mathrm{~d}$ following the lesion of anterior PS cortex in the left hemisphere.

to present cues in multiple locations throughout the visual field, allowed us to control the retinotopic locations of stimuli; to discourage postural orientation toward the correct location during the delay period, which could negate the mnemonic requirement; and also to analyze quantitatively more features of the monkey's behavioral responses (reaction time, response amplitude, response accuracy, etc.). These features of the ODR paradigm help to identify the possible causes for behavioral deficits, and to evaluate whether and to what degree they are attributable to degradation of memory processes, oculomotor control mechanisms, or visual perception.

The prefrontal cortex has been thought of as essential for motor set, initiation of or preparedness to respond (Fuster, 1989), and indeed, about $20 \%$ of the saccade-related activity recorded from the neurons of the PS region in monkeys performing an ODR task discharge prior to an oculomotor response (Funahashi et al., 1991). However, the behavioral deficits observed in the present study are not likely to be caused by interference with

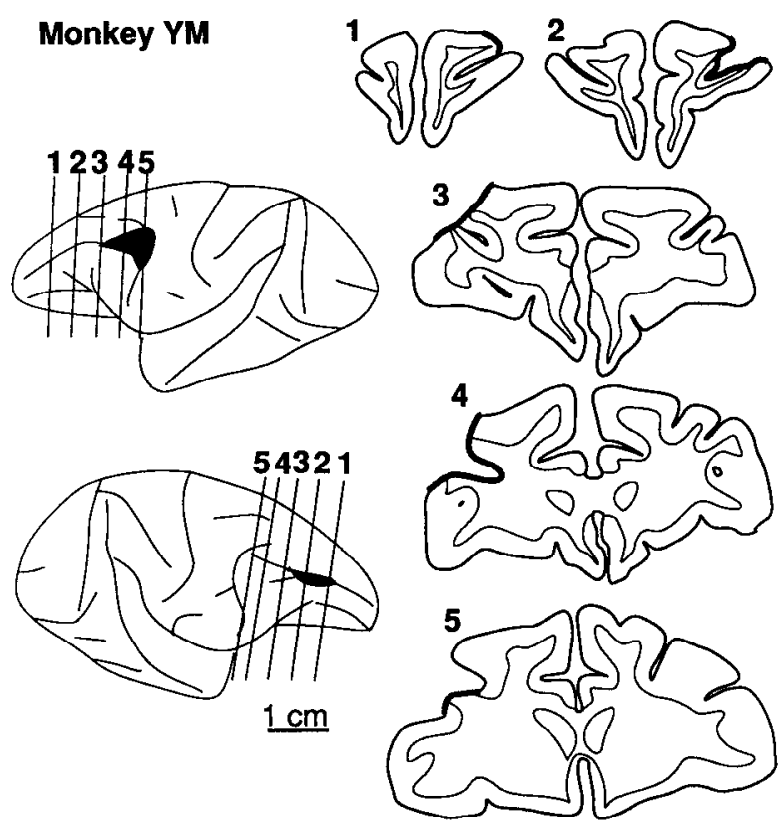

Figure 15. Reconstruction of the lesions in both hemispheres of monkey YM. The lesion in the left prefrontal cortex was located in the caudal one-third of the PS and also involved the anterior bank of the AS where the FEF is located. The subsequent lesion in the right prefrontal cortex was located in the middle third of the PS and spared the AS.

oculomotor control mechanisms. First, when the lesion was restricted to the PS region of cortex, reaction times were unaffected following either the first or second stage lesions, in spite of marked changes in accuracy measures across delay conditions. Second, saccadic velocities were the same before and after the lesions. Third, the trajectories of each saccadic eye movement made in the response period were similar across different lesion conditions, across different delay conditions, and across different task conditions. Finally, the monkeys performed normally on sensory-guided control tasks as well as when the delays were very brief, $1.5 \mathrm{sec}$. All of these results indicate that the oculomotor response mechanisms are intact after lesions involving the PS. In addition, the results are in accord with our physiological studies of oculomotor activity in monkeys performing the ODR task that show that the great majority (about $80 \%$ ) of saccade-related activity in PS neurons is postsaccadic; that is, neural activity begins for the most part after the initiation of the saccadic eye movements (Funahashi et al., 1991). Thus, it is not surprising that lesions incorporating the PS had little effect on reaction times, average velocities, and trajectories of saccadic eye movements in the ODR tasks.

A clear dissociation between memory-guided and sensoryguided responses in the present study also rules out a visual perceptual loss as the basis of the behavioral deficits observed. This was shown by the fact that the monkeys performed perfectly on the sensory control tasks as well as at the shortest (1.5 sec) delays in the ODR paradigm. As in the ODR paradigm, one of the control tasks required the monkey to delay its response but the sensory cue remained on during the delay so that a stimulus was present at the end of the delay to direct the response. Under this condition, performance was always accurate, even when the monkey was impaired in the memoryguided version that required the same responses (see Figs. 2, 5). 


\section{Monkey YM Task ODR-I \\ Before Lesion}
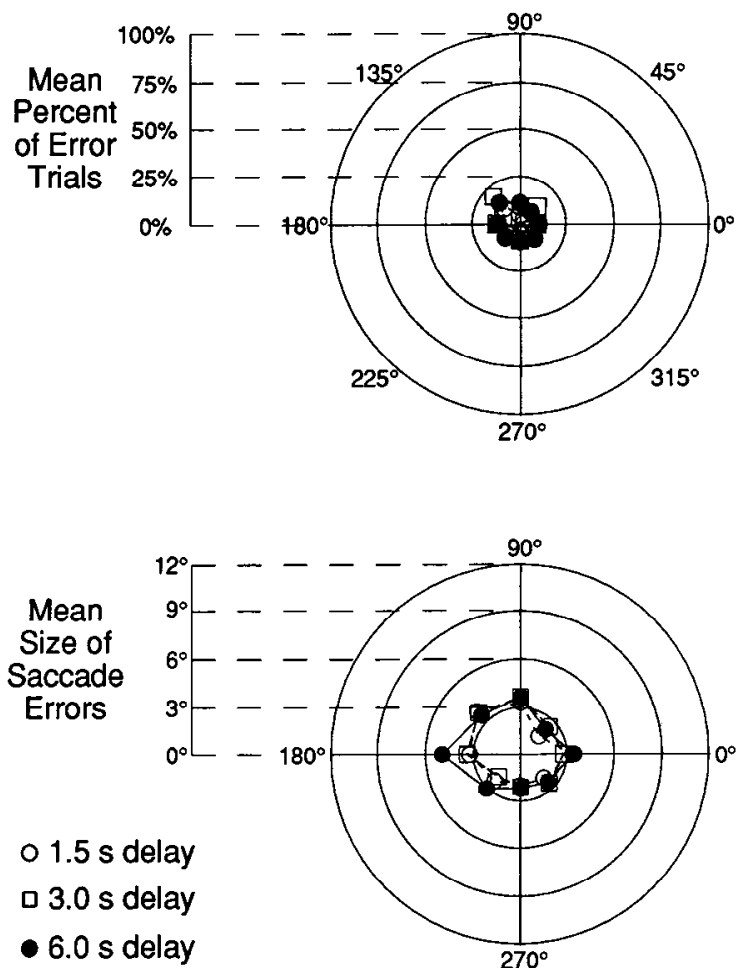
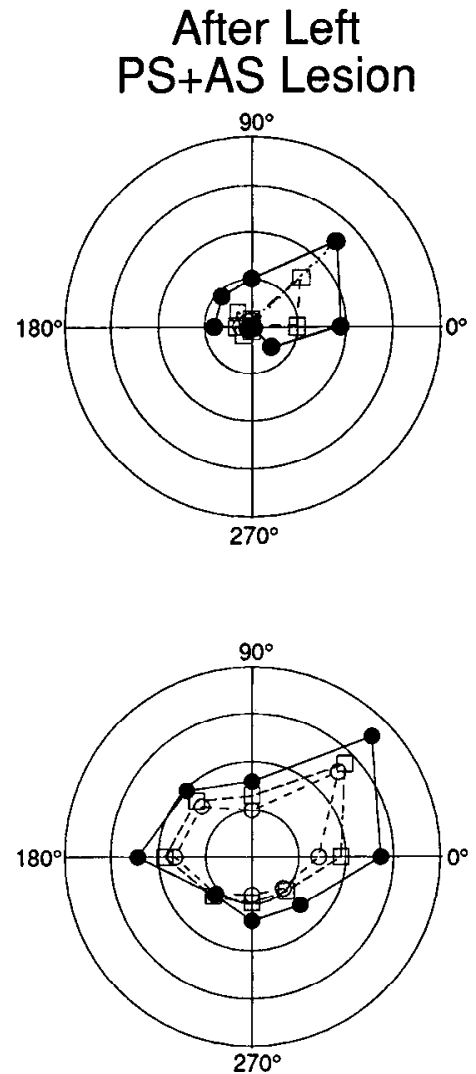

Plus Right PS Lesion
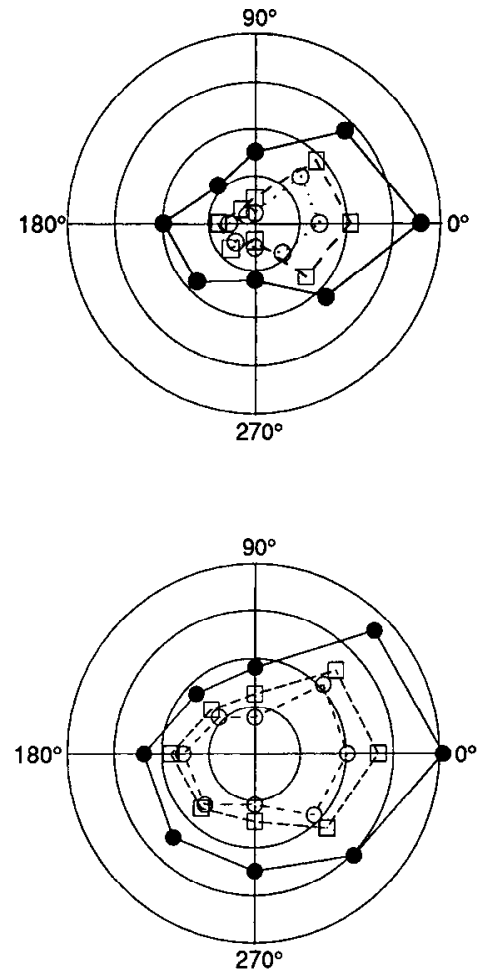

Figure 16. Summaries of ODR-I task performance of monkey YM before and after each of its lesions. The top row shows the percentages of trials on which the monkey's saccade fell outside the rewarded window, constituting an error trial. The bottom row shows the average sizc of the saccade error, that is, the distance (in degrees subtended) between where the cue was located and the end point of the saccade made toward the cue's remembered location. The left column shows both types of graphs for the average of three sessions conducted just before the lesion was made. The middle column averages sessions conducted 15,16 , and $17 \mathrm{~d}$ following the lesion of the left hemisphere. The right column averages sessions conducted 13,14, and $15 \mathrm{~d}$ following the lesion of the right hemisphere. Conventions are as in Figure 4. Note that following combined PS+AS lesion in the left hemisphere there was a substantial increase in both error percentage and error size for trials directed right and up $\left(45^{\circ}\right)$, and also for trials directed horizontally to the right $\left(0^{\circ}\right)$. Unlike deficits for lesions confined to the PS, these increased errors were for all three delay intervals.

A similar dissociation was recently demonstrated with intracerebral injections of the dopaminergic $D_{1}$ receptor antagonist SCH23390 directly into the PS. The local application of drug induced target-specific deficits on ODR trials but not on sensoryguided control trials that were introduced in the same session (Sawaguchi and Goldman-Rakic, 1991). Indeed, little evidence has ever been adduced that prefrontally lesioned animals have difficulty detecting visual cues, and, if anything, prefrontal lesions may heighten sensitivity to external stimuli (e.g., Konoski and Lawicka, 1964; Bartus and LeVere, 1977). The present finding is in keeping with the long tradition of research on this topic and serves to underscore its validity.

The most likely explanation for the behavioral deficits observed here is that they are caused by disruption or elimination of a transient spatial memory trace that holds the memory of a given spatial coordinate "on line" to direct the response at the end of the delay. This "on-line" memory process has been termed "working memory" (Goldman-Rakic, 1991), in analogy to the short-term storage mechanism of the same name in humans (Baddeley, 1986). In the present study of macaque monkeys, analyses of saccade accuracy and the distributions of saccadic end points showed that the poor performance of the lesioned monkeys in their contralateral visual field was based mainly on saccadic eye movements to the wrong directions. Similarly mis- directed eye movements were observed in both types of ODR task and only when the visual cues were presented in the contralateral visual field. Moreover, these erroneous or misguided responses were primarily made at longer ( 3 and $6 \mathrm{sec}$ ) delay periods. Similar results have been reported in previous studies examining the effects of cooling on the manual delayed-response task in a Wisconsin General Test Apparatus (e.g., Bauer and Fuster, 1976). These and other findings indicate that the monkey's problem in making correct responscs after a prefrontal lesion reflects a difficulty in retaining information about the cue location during the delay period and not by the loss of motor control or visual neglect or inattention within the restricted visual field.

This conclusion is further supported by our single-neuron recording studies using the same ODR task (Funahashi et al., $1989,1990,1991)$. These studies indicate that a substantial proportion of neurons in and around the PS had "memory fields"; that is, they exhibited tonic activation during the entire delay period of an ODR trial and only when the cue had been presented in a specific part of the visual field. Furthermore, different neurons code different target locations such that all parts of the visual field are represented in the population of neurons surrounding the PS. Finally, we have previously shown that when monkeys made errors, the delay-period activity of 


\section{Monkey YM Task ODR-I: $45^{\circ}$ and $225^{\circ}$ trials only}
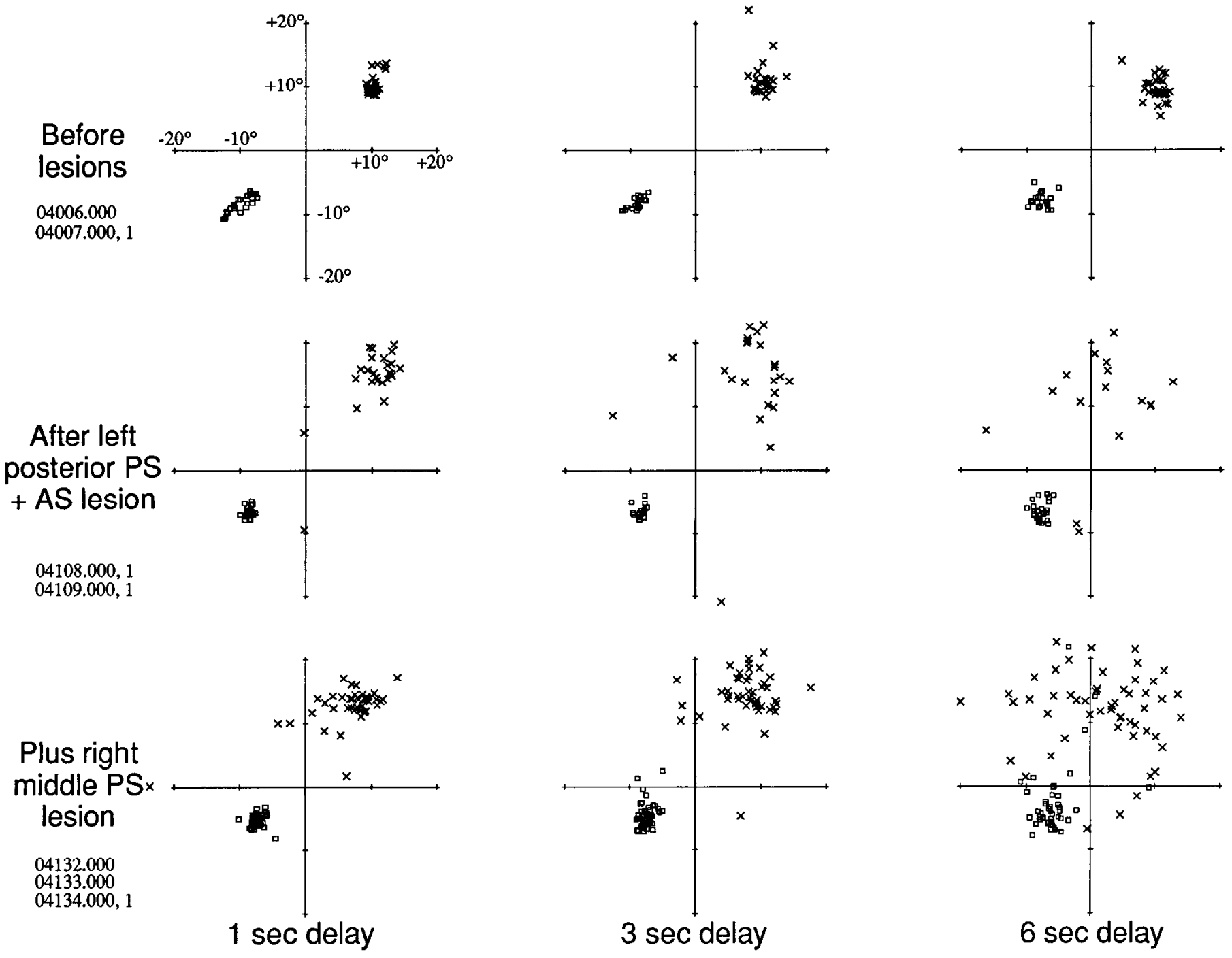

Figure 17. Saccadic end point distributions in the $45^{\circ}$ and $225^{\circ}$ trials of monkey YM on task ODR-I before lesions and after each of its lesions. Saccadic end points from trials with the cue having a $45^{\circ}$ direction (up and right) are plotted with $X$ 's, whereas trials in the opposite direction $\left(225^{\circ}\right.$, down and left) are plotted with boxes. The three delay period durations $(1.5,3$, and 6 sec) are plotted separately in the three columns. The top row of plots superimposes data from 2 consecutive days just before the first lesion. The middle row of plots superimposes data from 16 and 17 $\mathrm{d}$ following the lesion in the left hemisphere. The bottom row of plots superimposes data from 13, 14, and $15 \mathrm{~d}$ following the lesion in the right hemisphere. Notice that in the $45^{\circ}$ trials the overall scatter of saccadic end points, and in particular the number of saccades finishing outside the upper right quadrant, increased following the left hemisphere PS +AS lesion, especially for the 3 and 6 sec delay trials. Following the second lesion the $225^{\circ}$ trials were disrupted, most notably for the $6 \mathrm{sec}$ delay trials, and there was no apparent recovery for the $45^{\circ}$ trials.

prefrontal neurons was not maintained (see Fig. 13 in Funahashi et al., 1989). All of these considerations are consistent with a transient memory function of PS cortex.

\section{Laterality of spatial mnemonic function and the concept of a mnemonic "scotoma"}

Although impairments in the classical delayed-response tasks were originally observed in monkeys with bilateral ablations of large areas in the dorsolateral prefrontal cortex (Jacobsen, 1935), the present study indicates that a relatively small unilateral prefrontal lesion in the PS area is sufficient to produce moderate behavioral deficits in performance of ODR tasks. Only a few previous studies have reported delayed-response impairments with unilateral resections in the prefrontal cortex (Warren et al.,
1969; Warren and Nonneman, 1976). In these early studies, the magnitude of the behavioral deficit was always larger following bilateral lesions than after a unilateral lesion. Similar results have been obtained with the reversible lesion method of cryogenic depression wherein cooling the dorsolateral prefrontal cortex of one hemisphere produced more errors in a delayed-response task by the contralateral hand than the ipsilateral one (Fuster and Alexander, 1970). The present finding that the behavioral deficits in monkeys with unilateral lesions are largely restricted to the visual field contralateral to the lesioned hemisphere and appear only under conditions requiring memory guidance of responses makes a strong case for the mnemonic nature of these contralateral effects. Also, together with earlier studies, they show that the deficits transcend the effector system 
Figure 18. Saccadic end point distributions of monkey YM in the ODR-I task before lesions and after each of its lesions. The three delay period durations $(1.5,3$, and $6 \mathrm{sec})$ are plotted separately in the three rows. The eight cue positions (eccentricity $13^{\circ}$ ) are plotted with a small $x$ in each of the nine plots, and the eight ellipses in each plot convey overall saccade accuracy for each cue position. Each ellipse is centered at the mean $\mathrm{x}$ - and $\mathrm{y}$-coordinates of the saccadic end points for its cue, and the horizontal and vertical axes of the ellipses equal the SD of these $\mathrm{x}$ - and $y$-coordinates, respectively. The left column of plots shows data collected a few days before the first lesion was giv$\mathrm{cn}$. The middle column of plots shows data from $11 \mathrm{~d}$ following the lesion of PS cortex in the left hemisphere. The right column shows data from $13 \mathrm{~d}$ following the subsequent lesion of PS cortex in the right hemisphere.
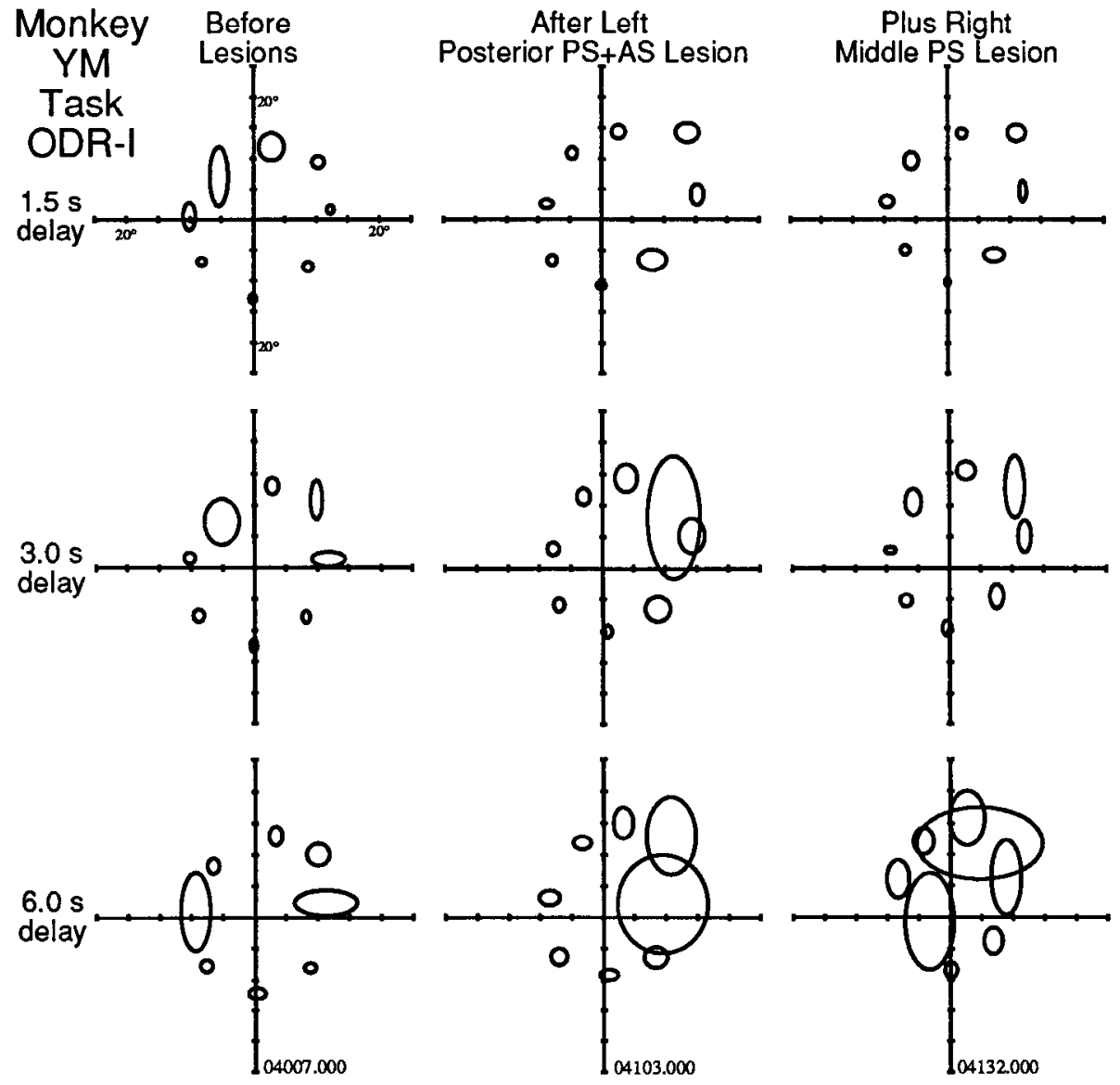

used to assess the animal's working memory capacity, and indicate why bilateral lesions have often been necessary to reveal the spatial-mnemonic deficit in traditional testing situations. For example, monkeys tested in a Wisconsin General Test Apparatus are free to move around and could easily compensate for the restricted locus of stimulus presentation when the head is fixed.

The control of retinal coordinates of cue locations and hence precise control of the targets encoded in working memory in the ODR task allowed the discovery that the mechanisms subserving memory-guided behavior are organized within a retinotopic, egocentric framework and are lateralized in much the same way as the visual world is represented in the visual cortices; that is, the behavioral impairments observed were consistently more severe when the visual cues were presented in the visual field contralateral to the hemisphere than when they were presented in the ipsilateral visual field. Thus, it appears that the PS in the left hemisphere is concerned with remembering information about the right visual field and the right PS is specialized for memories of left visual field locations. Furthermore, the unilateral lesions rarely uniformly affected memory for targets in all parts of the visual field. Most of the impairments observed were more severe for stimuli presented in one or another quadrant or in one polar direction. In analogy with visual field defects observed after occipital cortical lesions, and in order to emphasize their retinotopic organization, we refer to these partial mnemonic field deficits generally as mnemonic scotomas. They could equally be designated mnemonic hemianopias or mnemonic quadrantanopias, depending upon the extent of contralateral field involvement.

These findings are supported by additional work. The injection of pharmacological agents by syringe into small (approximately $2 \mathrm{~mm}^{3}$ ) volumes of cortex has likewise shown that the memory deficit can be circumscribed even to a particular contralateral target location (Sawaguchi and Goldman-Rakic, 1991). Additionally, at the cellular level, the memory fields, that is, directional delay-period activity of individual prefrontal neurons recorded from the PS area, are preferentially tuned for targets presented in the contralateral field (Funahashi et al., 1989). Thus, about $50 \%$ of fully characterized prefrontal neurons with directional delay-period activity have memory fields centered in the visual field contralateral to the hemisphere where the neurons are recorded, whereas only $17 \%$ have ipsilateral memory fields, and $17 \%$ and $16 \%$ have memory fields in upward and downward directions, respectively (Funahashi et al., 1989). Thus, although the population of neurons in each hemisphere may be capable of coding cue locations over the entire visual field, PS neurons within each hemisphere code mainly contralateral locations. The predominance of contralateral memory fields in these neurons presumably is the neural basis for the mainly contralateral behavioral deficits following unilateral PS lesions found in the present study.

\section{Effects of serial lesions on behavioral deficits}

Two monkeys in the present study were examined after an initial lesion and then again following a second lesion in the contra- 


\title{
Saccadic Reaction Times in Task ODR-I
}
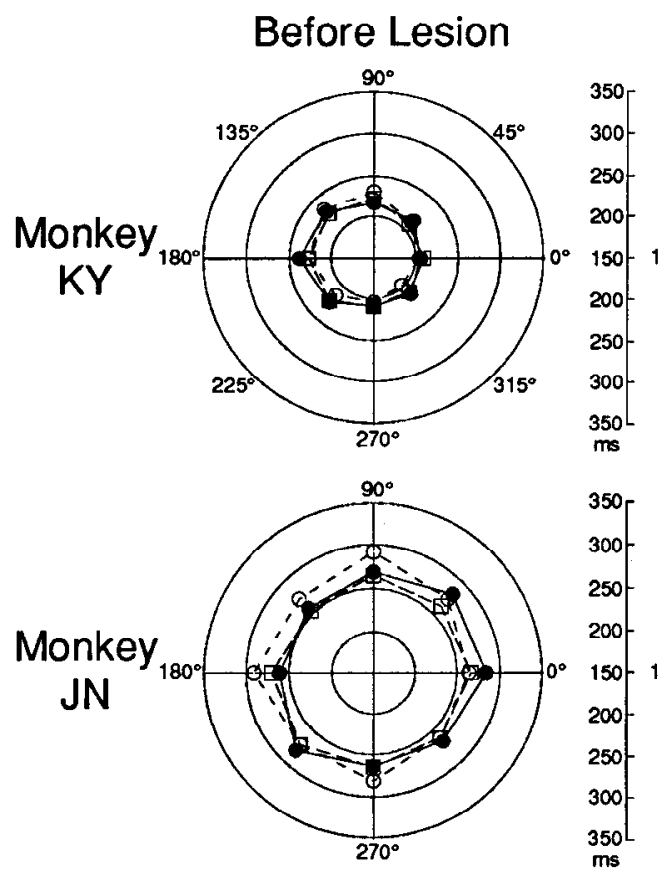

\author{
After Left PS Lesion
}

Plus Right PS Lesion

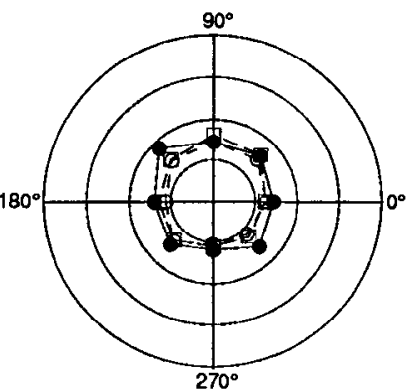

\section{No Second Lesion}
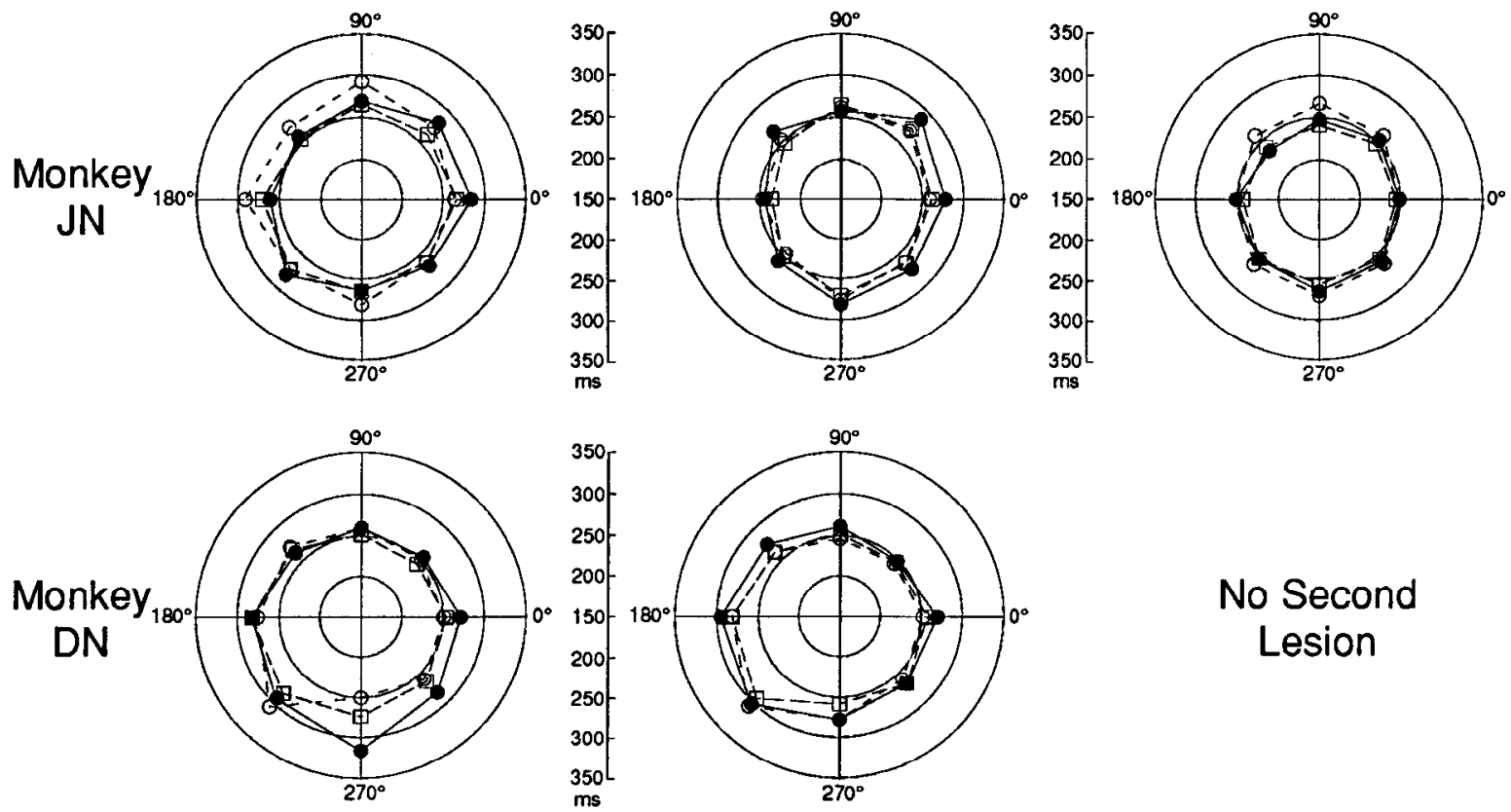

\section{No Second Lesion}
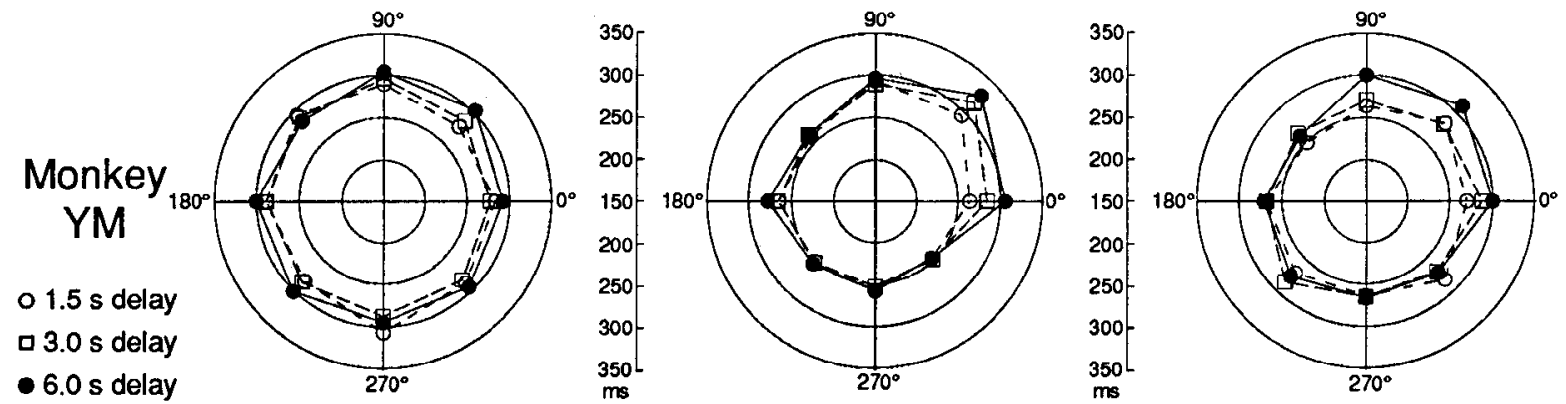

Figure 19. Mean saccadic reaction times in the ODR-I task for all four monkeys. These polar plots show the mean saccadic reaction time from two to four sessions conducted shortly before and shortly after the lesions were made. Saccadic reaction time was defined as the time interval between the disappearance of the fixation target and the initiation of the saccade. Each point on the polar plots corresponds to the monkey's mean saccadic reaction time at each cue direction/delay period combination (monkey DN's delay times were 1,2 , and 4 sec and thus slightly shorter than those of the other monkeys). Notice that saccadic reaction times were largely unaffected by the lesions, except for monkey YM's first lesion.

lateral PS. One of these monkeys (JN), following a second-stage right PS lesion, showed an immediate and striking recovery from its severe right hemifield deficit caused by its initial lesion of the left PS. This recovery was especially evident in the saccadic end point plots for the $6 \mathrm{sec}$ delay condition shown in Figure 9. It is reminiscent of the "Sprague effect" (e.g., Sprague, 1966) wherein a unilateral lesion of the cat's superior colliculus effected a quick recovery from hemineglect caused by a neocortical lesion made in the opposite hemisphere.

That substantial recovery from PS lesions can occur is not surprising since physiological studies have found delayed-response activity in several brain structures additional to the PS including the FEFs, the supplementary eye fields, the lateral intraparietal area, and the substantia nigra pars reticulata (for 


\section{Average Saccadic Velocities in Task ODR-I}
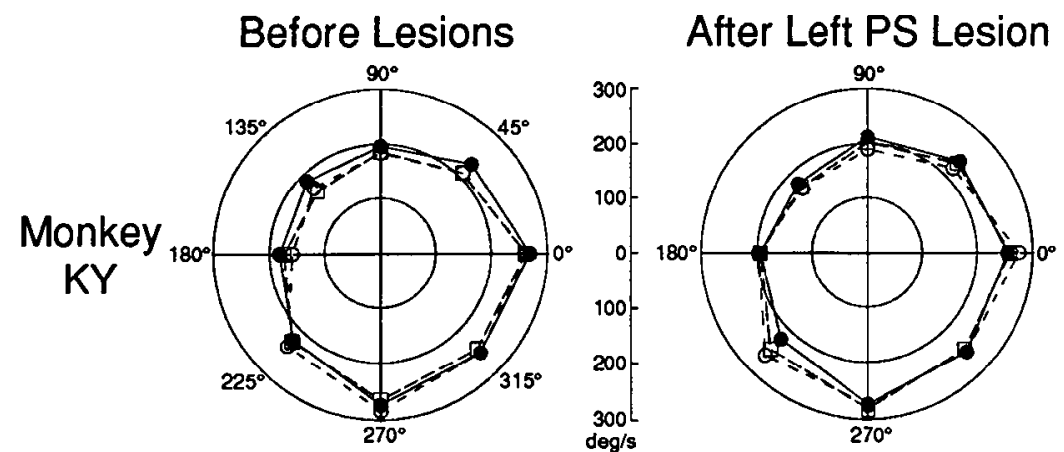

Plus Right PS Lesion
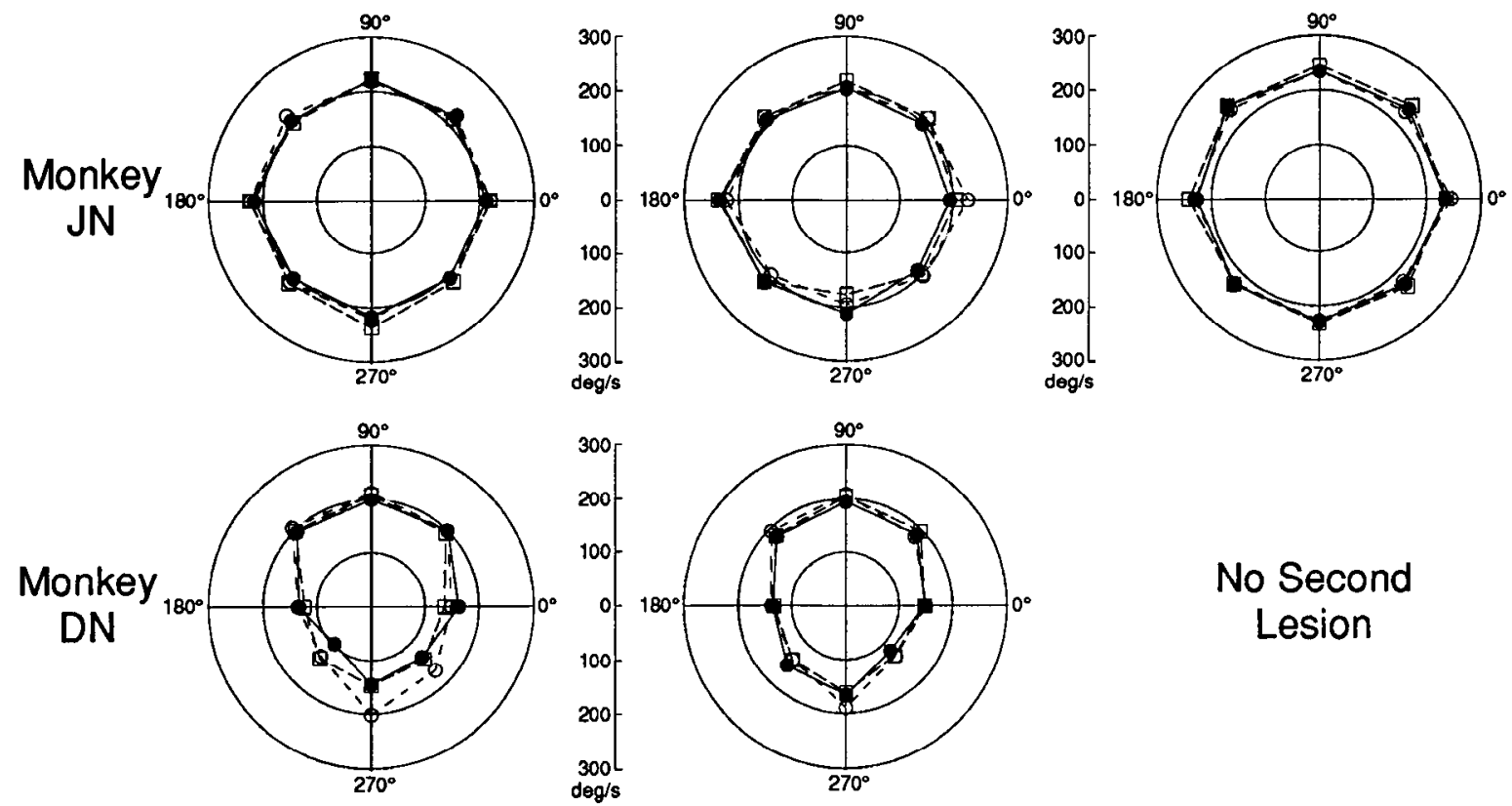

\section{No Second \\ Lesion}
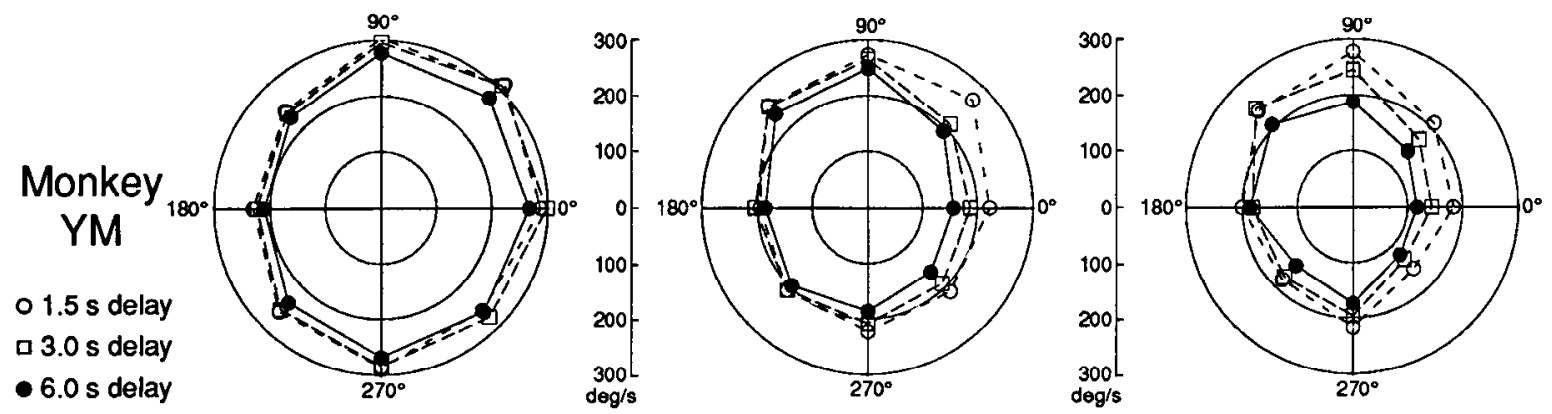

Figure 20. Average saccadic velocities in the ODR-I task for all four monkeys. These polar plots show data from two to four sessions conducted shortly before and shortly after the lesions were made (the same sessions as used for Figure 19 plots of saccadic reaction times). The average velocity of each saccade was calculated as saccade amplitude divided by saccade duration, and thus is much less than peak saccadic velocity. Each point plotted corresponds to the mean of the monkey's average saccadic velocity at each cue direction/delay period combination (monkey DN's delay times were 1, 2, and $4 \mathrm{sec}$ and thus slightly shorter than those of the other monkeys). Notice that average saccadic velocities were largely unaffected by the lesions, except for monkey YM's first lesion.

review, see Goldman-Rakic et al., 1992). However, in all of these structures, as in the PS, such activity is primarily for cues from the contralateral hemifield. We speculate that a unilateral PS lesion creates a tonic interhemispheric imbalance of activity in these related structures, favoring activity in the hemisphere with the intact PS and effectively inhibiting activity in the hemisphere that lacks a PS. In this asymmetrical situation, neuronal activity representing cucs in the hemificld contralateral to the lesion would dissipate more quickly than normal, resulting in more errors for longer delays. A hypothesis that could be tested, therefore, is that a subsequent PS lesion in the other hemisphere remedies this imbalance, and thus allows non-PS structures ipsilateral to the original lesion to function normally or more effectively. 


\section{Functional differences between the PS and FEF}

Changes in eye movement parameters (i.e., saccadic reaction times and average saccadic velocities) were observed only when the FEF was included in the lesion. Although only one monkey (YM) had an FEF lesion, our results are in basic agreement with the finding of Deng et al. (1987) that monkeys with unilateral FEF lesions are impaired in the acquisition of an ODR task with short (100 msec) delays). In the present study, the lesion that included the FEF produced a significant increase in saccadic reaction times, decrease in saccadic velocities, and longer-lasting behavioral deficits even at the shortest delay. Such changes in saccadic latencies and velocities were observed only when the monkey made eye movements directed toward the visual field contralateral to the hemisphere with the lesion. Deng et al. also found an increase of saccadic reaction times and a decrease of saccadic velocities when the monkey made saccades directed contralateral to the hemisphere with the FEF lesion. Unlike the PS, the FEF has been shown to be important in the control of purposive saccadic eye movements by lesion studies (Schiller et al., 1980; Deng et al., 1987) as well as neurophysiological studies (Bruce and Goldberg, 1985; Bruce, 1990). Moreover, microstimulation elicits saccadic eye movements at low thresholds in the FEF (Bruce et al., 1985; Huerta et al., 1987) but not in the PS. Thus, there can be little doubt that FEF participates more directly in oculomotor control than does the PS, which may be more involved in mnemonic coding and important for rclaying spatial information to motor centers, including the FEF.

\section{References}

Baddeley A (1986) Working memory. New York: Oxford UP. Bartus RT, Levere TE (1977) Frontal decortication in rhesus monkeys: a test of the interference hypothesis. Brain Res 119:233-248.

Bauer RH, Fuster JM (1976) Delayed-matching and delayed-response deficit from cooling dorsolateral prefrontal cortex in monkeys. J Comp Physiol Psychol 90:293-302.

Bruce CJ (1990) Integration of sensory and motor signals for saccadic eye movements in the primate frontal eye fields. In: Signal and sense, local and global order in perceptual maps (Edelman GM, Gall WE, Cowan WM, eds), pp 261-314. New York: Wiley.

Bruce CJ, Goldberg ME (1985) Primate frontal eye fields. I. Single neurons discharging before saccades. J Neurophysiol 53:606-635.

Bruce CJ, Goldberg ME, Bushnell MC, Stanton GB (1985) Primate frontal eye fields. II. Physiological and anatomical correlates of electrically evoked eye movements. J Neurophysiol 54:714-734.

Butters N, Pandya D (1969) Retention of delayed-alternation: effect of selective lesions of sulcus principalis. Science 165:1271-1273.

Butters N, Pandya D, Stein D, Rosen J (1972) A search for the spatial engram within the frontal lobes of monkeys. Acta Neurobiol Exp 32: 305-329.

Deng S-Y, Goldberg ME, Segraves MA, Ungerleider LG, Mishkin M (1987) The effect of unilateral ablation of the frontal eye fields on saccadic performance in the monkey. In: Adaptive processes in visual and oculomotor systems (Keller EL, Zee DS, eds), pp 201-208. New York: Pergamon.

Funahashi S, Bruce CJ, Goldman-Rakic PS (1986) Perimetry of spatial memory representation in primate prefrontal cortex: evidence for mnemonic hemianopia. Soc Neurosci Abstr 12:554.

Funahashi S, Bruce CJ, Goldman-Rakic PS (1989) Mnemonic coding of visual space in the monkey's dorsolateral prefrontal cortex. J Neurophysiol 61:331-349.
Funahashi S, Bruce CJ, Goldman-Rakic PS (1990) Visuospatial coding in primate prefrontal neurons revealed by oculomotor paradigms. J Neurophysiol 63:814-831.

Funahashi S, Bruce CJ, Goldman-Rakic PS (1991) Neuronal activity related to saccadic eye movements in the monkey's dorsolateral prefrontal cortex. J Neurophysiol 65:1464-1483.

Fuster JM (1989) The prefrontal cortex. New York: Raven.

Fuster JM, Alexander GE (1970) Delayed response deficit by cryogenic depression of frontal cortex. Brain Res 20:85-90.

Goldman PS, Rosvold HE (1970) Localization of function within the dorsolateral prefrontal cortex of the rhesus monkey. Exp Neurol 27: 291-304.

Goldman PS, Rosvold HE, Vest B, Galkin TW (1971) Analysis of the delayed-alternation deficit produced by dorsolateral prefrontal lesions in the rhesus monkey. J Comp Physiol Psychol 77:212-220.

Goldman-Rakic PS (1987) Circuitry of primate prefrontal cortex and regulation of behavior by representational memory. In: Handbook of physiology, Sec 1, The nervous system, Vol V (Plum F, ed), pp 373417. Bethesda, MD: American Physiological Society.

Goldman-Rakic PS (1990) Cellular and circuit basis of working memory in prefrontal cortex of nonhuman primates. Prog Brain Res 85 . 325-326.

Goldman-Rakic PS, Chafee M, Friedman H (1992) Allocation of spatial function in distributed circuits. New York: Oxford UP, in press.

Huerta MF, Krubitzer LA, Kaas JH (1987) Frontal eye field as defined by intracortical microstimulation in squirrel monkeys, owl monkeys, and macaque monkeys. II. Cortical connections. J Comp Neurol 265 : 332-361.

Jacobsen CF (1935) An experimental analysis of the frontal association areas in primates. Arch Neurol Psychiatry 33:558-569.

Judge SJ, Richmond BJ, Chu FC (1980) Implantation of magnetic search coils for measurement of eye position: an improved method. Vision Res 20:535-538.

Konorski J, Lawicka W (1964) Analysis of errors by prefrontal animals on delayed-response test. In: The frontal granular cortex and behavior (Warren JM, Akert K, eds), pp 271-312. New York: McGraw-Hill.

Luria AR (1966) Higher cortical functions in man. New York: Basic Books.

Park S, Holzman P (1992) Schizophrenics show spatial working memory deficits. Arch Gen Psychiatry, in press.

Robinson DA (1963) A method of measuring eye movement using a scleral search coil in a magnetic field. IEEE Trans Biomed Eng 10: 137-145.

Rosenkilde CE (1979) Functional heterogeneity of the prefrontal cortex in the monkey: a review. Behav Neural Biol 25:301-345.

Sawaguchi T, Goldman-Rakic PS (1991) D1 dopamine receptors in prefrontal cortex: involvement in working memory. Science 251:947950.

Schiller PH, True SD, Conway JL (1980) Deficits in eye movements following frontal eye-field and superior colliculus ablations. J Neurophysiol 44:1175-1189.

Sprague JM (1966) Interaction of cortex and superior colliculuc in mediation of visually guided behavior in the cat. Science 53:1544 1547.

Teuber H-L (1972) Unity and diversity of frontal lobe functions. Acta Neurobiol Exp 32:615-656.

van Gisbergen JAM, Robinson DA, Gielen SA (1981) A quantitative analysis of generation of saccadic eye movements by burst neurons. J Neurophysiol 45:417-442.

Walker AE (1940) A cytoarchitectural study of the prefrontal area of the macaque monkey. J Comp Neurol 73:59-86.

Warren JM, Nonnemann AJ (1976) The search for cerebral dominance in monkeys. Ann NY Acad Sci 280:732-744.

Warren JM, Cornwell PR, Warren HB (1969) Unilateral frontal lesions and learning by rhesus monkeys. J Comp Physiol Psychol 69:498505 . 